\title{
Cartographier les mouvements migratoires
}

Mapping the Migratory Movements

Cartografiar los movimientos migratorios

Lucie Bacon, Olivier Clochard, Thomas Honoré, Nicolas Lambert, Sarah Mekdjian et Philippe Rekacewicz

\section{CpenEdition}

Journals

Édition électronique

URL : https://journals.openedition.org/remi/8249

DOI : $10.4000 /$ remi.8249

ISSN : $1777-5418$

Traduction(s) :

Mapping the Migratory Movements - URL : https://journals.openedition.org/remi/8803 [en]

Éditeur

Université de Poitiers

Édition imprimée

Date de publication : 1 décembre 2016

Pagination : 185-214

ISBN : 979-10-90426-29-0

ISSN : 0765-0752

Référence électronique

Lucie Bacon, Olivier Clochard, Thomas Honoré, Nicolas Lambert, Sarah Mekdjian et Philippe

Rekacewicz, "Cartographier les mouvements migratoires », Revue européenne des migrations

internationales [En ligne], vol. 32 - n³ et 4 | 2016, mis en ligne le 01 décembre 2018, consulté le 14 avril 2022. URL : http://journals.openedition.org/remi/8249; DOI : https://doi.org/10.4000/remi.8249 


\title{
Cartographier
}

\section{les mouvements migratoires}

\section{Lucie Bacon ${ }^{1}$, Olivier Clochard ${ }^{2}$, Thomas Honoré ${ }^{3}$, Nicolas Lambert ${ }^{4}$, Sarah Mekdjian ${ }^{5}$ et Philippe Rekacewicz ${ }^{6}$}

\begin{abstract}
Cartographier les mouvements migratoires, c'est-à-dire nécessairement " immobiliser " un système spatio-temporel, social, politique complexe, relève d'un véritable défi. Non seulement les individus en migration et/ou sur le chemin de l'exil se croisent, mais ils font aussi des "pauses ", plus ou moins longues, ils s'installent temporairement dans un pays, dans un lieu, y restent quelques jours, quelques semaines ou quelques années et parfois en repartent. La géographie et la cartographie des migrations sont soumises au temps et à des évolutions politiques qui peuvent être très rapides. Ainsi, la cartographie risque toujours d'être anachronique avant même que la réalisation de la carte ne soit terminée. Par ailleurs, la mesure des "flux ", dont l'étymologie latine fluxus rappelle son sens premier d' " écoulement ", suppose d'analyser les migrations en agrégeant des données, à la fois spatiales et temporelles, avec des traitements qui peuvent être très différents. Le terme général de "flux ", qui sert à qualifier de nombreux titres de cartographies migratoires, est rarement explicité. Ainsi, un flux migratoire peut être mesuré à partir du comptage du nombre de passages, en un lieu, effectués pendant un intervalle de temps donné (par exemple, le nombre de personnes ayant traversé la frontière États-Unis-Mexique, en 2015, à Ciudad Juárez) ; il peut aussi être mesuré entre deux points (le nombre de personnes étant parties du Chiapas au Mexique et s'étant rendu en Californie en 2015). Dans le premier cas, il s'agit d'un comptage du mouvement en train de se faire
\end{abstract}

\footnotetext{
1 Géographe, doctorante, Université de Poitiers, MIGRINTER, MSHS, Bâtiment A5, 5 rue Théodore Lefebvre,TSA 21103, 86073 Poitiers cedex 9 ; lucie.bacon.bih@gmail.com

2 Géographe, chargé de recherche au CNRS, membre de Migreurop, Université de Poitiers, MIGRINTER, MSHS, Bâtiment A5, 5 rue Théodore Lefebvre, TSA 21103, 86073 Poitiers cedex 9 ; olivier.clochard@univ-poitiers.fr

3 Cartographe, inCittà, 5 avenue André Bacon, 13013 Marseille ; thomas.honore82@ gmail.com

4 Cartographe, membre de Migreurop, Université Paris Diderot, RIATE, Bâtiment Olympe de Gouges, 8 place Paul Ricœur, 75013 Paris ; nicolas.lambert@ums-riate.fr

5 Géographe, enseignante-chercheuse, Université Grenoble Alpes, PACTE, Institut d'études politiques, BP 48, 38040 Grenoble cedex 9 ; sarah.mekdjian@univ-grenoblealpes.fr

6 Cartographe, Visionscarto ; rekacewi@online.no
} 
et mesuré à un endroit précis ; dans le second, le mouvement est reconstitué a posteriori à partir de données quantitatives absolues (le "stock " de personnes mexicaines du Chiapas recensées en Californie depuis 2015). Les cartes prennent aussi en charge des mouvements individuels, qui ne sont alors plus appelés "flux ", mais " itinéraires " ou " parcours ", en croisant des données spatiales à des données qui peuvent être sociales, politiques, temporelles ${ }^{7}$. Rendre compte de la complexité de données temporelles à partir de la cartographie, qui relève d'une analogie spatiale, est un horizon de recherche qui a donné lieu à des innovations techniques et épistémologiques, notamment les chorèmes statiques, puis dynamiques, les cartographies informatiques animées dont certaines sont interactives (Kaddouri, 2008).

En plus du défi de la représentation du mouvement, l'accessibilité des données statistiques peut également être difficile dans le domaine des études migratoires. Les instruments statistiques dont nous disposons ${ }^{8}$ sont souvent incomplets, imprécis, voire critiquables, dans leur mode de construction. II faut néanmoins noter de grands progrès qualitatifs, depuis les années 2000, stimulés notamment par le développement du data journalism ${ }^{9}$. Pour cartographier les processus migratoires, et ce à différentes échelles, il est nécessaire de " généraliser " et de traiter les données disponibles en " paquets ", c'est-à-dire de synthétiser les informations disponibles pour imaginer les représentations de grandes tendances, à défaut d'être capable de les quantifier précisément. En complément de ce travail, nombre de chercheurs travaillent sur le terrain et utilisent la cartographie " à fine échelle " comme un outil d'analyse destiné à mieux comprendre les processus en cours dans les camps, les lieux d'accueil, les gares, les aéroports, les villes et leurs quartiers. Des initiatives participatives, ouvertes à la société civile, en dehors des uniques cercles académiques, comme Close the camps ${ }^{10}$, Migrants files ou encore Watch the Med, permettent de mettre en place un appareil de collecte de données quantitatives et qualita-

\footnotetext{
7 Voir par exemple la carte " De Kaboul à Calais, l'itinéraire de Khan " imaginée et réalisée parThomas Honoré et Emmanuelle Hélio (Migreurop, 2012).

8 Parmi les nombreuses bases de données statistiques que nous utilisons pour créer des cartes de flux migratoires à différentes échelles, il y a celles d'Eurostat, du HautCommissariat des Nations unies pour les réfugiés (UNHCR), de I'Organisation de coopération et de développement économiques (OCDE), de I'Institut national d'études démographiques (INED), etc.

9 Contrairement au journalisme traditionnel qui ne divulgue pas ou très peu ses sources, le data journalism indique les données utilisées et les rend accessibles à travers des outils de visualisation (par exemple, OWNI : http://owni.fr/tag/data-journalisme/; The Migrants' Files : http://www.themigrantsfiles.com/).

10 http://closethecamps.org/
} 
tives et ce, grâce à des personnes qui recueillent des informations disparates ${ }^{11}$. Ces modes de production de la connaissance, encore en cours d'élaboration, $\mathrm{s}^{\prime}$ avèrent particulièrement prometteurs.

À la prise en compte des temporalités dans la cartographie migratoire, et à la nécessité de recueillir des données vérifiables, s'ajoutent des enjeux représentationnels cartographiques spécifiques. "Message dessiné " qui peut être fictionnel et/ou référentiel, la carte est un iconotexte (Cosgrove, 2001 : 148), un ensemble de symboles graphiques, définis par des attributs de formes, de tailles, de couleurs, auxquels on attribue une signification : des flèches, des carrés, des ronds ou des traits qui, sur la carte, symbolisent des réfugiés, des camps, des points de passages, des murs ou des frontières. La légende explicative des éléments constitutifs de la carte répond à une grammaire et une sémiologie singulières. Les cartes de flux migratoires, très souvent élaborées à partir du symbole de la flèche, sont les modèles les plus connus de figuration et de formalisation de données spatio-temporelles relatives aux migrations. Dans le cas des cartes référentielles, le passage des réalités observées à la représentation symbolique, en particulier par la flèche, n'est pas sans danger. Tous les choix graphiques - nécessairement subjectifs, même si inspirés de langages institués, comme la sémiologie graphique de Bertin (1967) - peuvent conduire à des interprétations en décalage, voire en contradiction avec les intentions des cartographes : des flèches trop épaisses qui pointent dans la même direction risquent d'évoquer (ou suggèrent plus ou moins inconsciemment) " une invasion "; des ronds trop petits, à peine visibles, peuvent constituer un déni de reconnaissance d'une importante population réunie dans un camp. À partir d'une étude de cartes médiatiques des migrations transsahariennes, Choplin et Pliez (2011) ont ainsi critiqué, la construction " d'un espace migratoire lisse, c'est-à-dire où le trait de dessin continu de quelques routes migratoires occulte toutes les "aspérités" - spatiales et temporelles d'ordre politique, policier, pécuniaire... qui jalonnent les itinéraires empruntés par les migrants ". En effet, un des défis de la cartographie des migrations, en complément des approches quantitatives et agrégées de ces mobilités, est de rendre compte du mouvement dans ses dimensions qualitatives et sensibles, notamment depuis le point de vue de celles et ceux qui se déplacent. Les deux auteurs, qui soulignent ici une tendance des cartes migratoires au réductionnisme, à la déshumanisation et à la dépolitisation

11 Par exemple, la carte des "Morts aux frontières ", imaginée en 2002 par Olivier Clochard, aurait été impossible à établir sans le travail des membres de l'ONG UNITED à Amsterdam et de leur réseau, qui ont, les premiers, essayé de documenter les décès de migrants dès les années 1990. Ils ont été rejoints par la suite par des initiatives personnelles comme celles du journaliste Gabriele Del Grande (voir son site : http://fortresseurope.blogspot.fr/) et plus récemment par des institutions comme l'Organisation internationale pour les migrations (OIM) et le Haut-Commissariat des Nations unies pour les réfugiés (UNHCR). Depuis 2013, le projet The Migrant Files (http://www.themigrantsfiles. $\mathrm{com} /$ ) - ouvert et participatif - réunit et croise les statistiques de ces différentes bases de données enregistrées depuis 2000. Avec des mises à jour régulières, ces " producteurs de données " sont aussi des acteurs très importants de la "fabrication " de la carte. Car les travaux cartographiques des acteurs non gouvernementaux et individuels sont aussi très fragiles, pouvant s'arrêter du jour au lendemain faute de moyens humains ou financiers pour continuer, nous laissant orphelins d'informations souvent cruciales et inexistantes par ailleurs. Pour rappel, la première version de la carte des " Morts aux frontières de I'Union européenne " est parue dans la revue Les cahiers d'outre-mer (Clochard, 2003). Elle a ensuite été retravaillée avec Philippe Rekacewicz, et publiée en 2004 dans Le Monde diplomatique. Elle a, depuis, été de nombreuses fois actualisée et complétée. 
des contextes de déplacements, notent aussi, pour certaines " cartes médiatiques ", le risque d'une confusion entre " itinéraires " et "flux ", alimentant encore l'angoisse de l'« invasion ": "Les longs traits qui figurent la migration africaine vers l'Europe restituent l'image un peu inquiétante d'une invasion passant par des itinéraires (les villes de Ceuta et Melilla, la Libye, etc.) qui sont pourtant rarement empruntés simultanément par des milliers de migrants. De telles cartes font oublier que ces flux sont marginaux au regard des migrations africaines et même des migrations transsahariennes " (Choplin et Pliez, 2011).

L'européocentrisme, ou plus largement la reproduction d'une division entre un " nord " et un " sud ", le choix de fonds de carte normés qui reconduisent I'imaginaire politique de frontières linéaires et fixes, la réduction du mouvement à des flèches ou à des mesures de stocks, sont autant d'enjeux scientifiques, représentationnels et politiques posés à la cartographie des mouvements migratoires.

Un autre risque posé par la cartographie migratoire relève des usages qui en sont faits par les dispositifs étatiques et supra-étatiques pour surveiller et tracer les mouvements de populations et d'individus. Les systèmes d'information géographique, les GPS, et la cartographie en général, sont, en effet, des outils mis au service de politiques de contrôle et d'exclusion. Dans le registre scientifique, il est donc important de s'interroger sur les usages possibles des cartes produites, hors du monde académique, et sur les liens entre production du savoir et politique. C'est bien ici toute une éthique du travail de représentation qui est en jeu, en lien avec des contextes politiques et idéologiques conflictuels.

Ainsi, à partir d'une analyse de l'histoire et de l'actualité de la cartographie des flux migratoires, issue de sources très diverses - scientifiques, artistiques, militantes - et de différents contextes continentaux, régionaux et nationaux, nous proposons d'analyser quelques-uns des principaux défis techniques, éthiques, institutionnels et politiques que pose la représentation cartographique des migrations internationales. Dans une perspective critique, nous tenterons d'analyser à la fois le rôle de la cartographie dans la production du savoir et de la connaissance dans le domaine migratoire, mais aussi son potentiel de transformation sociale et politique.

Dans un premier temps, nous traçons quelques lignes structurantes de l'évolution de la cartographie scientifique relative aux mouvements migratoires, depuis ses débuts au XIXe siècle jusqu'à aujourd'hui, pour identifier et analyser, dans un second temps, les principaux défis techniques et épistémologiques, et les enjeux politiques qu'elle continue de poser. Enfin, nous présentons une typologie de cartographies migratoires créatives et indisciplinaires, entre science, art et militantisme.

\section{Représentation des flux migratoires : évolutions et enjeux}

C'est au XIXe siècle, avec la naissance de la cartographie thématique moderne utilisant des statistiques démographiques, sociologiques, économiques, qu'émergent les premières cartes de flux migratoires. Cette cartographie se généralise néanmoins bien plus tardivement, à la fin des années 1980, quand les migrations s'instituent comme objets de recherche. 


\section{Des migrations mais peu de cartes pour les représenter}

Selon Bahoken (2013 : 2), la carte " Currents of Migrations " éditée en 1885, et réalisée " par Ernst Georg Ravenstein, géographe et cartographe allemand de la Royal Geographic Society (RGS) de Londres, [...] semble être la première carte des flux réalisée avec des flèches ". Cette carte n'établit pas de hiérarchisation entre les flux, elle relève $d^{\prime}$ 'une approche qualitative des mobilités ${ }^{12}$ internes au Royaume-Uni, à la Grande-Bretagne et à I'Irlande. Une autre carte réalisée au XIXe siècle ${ }^{13}$ montre les migrations d'ouvriers agricoles en Russie d'Europe vers les régions méridionales, jusqu'à la mer Noire et la mer Caspienne.

Alors que les migrations internationales au XIXe siècle sont marquées par des flux très importants ${ }^{14}$, et que la cartographie statistique a connu des avancées notoires entre 1835 et 1855 avec " différentes techniques de représentation [...] parmi lesquelles celle des mouvements " (Robinson, $1955: 1$ ), les cartes migratoires restent néanmoins très peu nombreuses. Seule la carte de Charles-Joseph Minard représente, de manière approximative et sans flèches, les migrations à l'échelle du globe en 1858 (cf. Carte 1) 1$)^{15}$.

En parcourant différents ouvrages et atlas publiés au tournant des XIXe et $\mathrm{XXe}$ siècles ${ }^{16}$, quelques documents - $\mathrm{s}^{\prime}$ intéressant à des processus plus localisés - utilisent des flèches comme éléments cartographiques. Nous pouvons citer en exemple les cartes de René Avelot imaginées en 1905 et dessinant les principales migrations au Gabon et au Congo français, ainsi que celle de Jovan Cvijić, publiée en 1918, et représentant les déplacements de populations peuplant en partie les pays serbes du XVe au début du XXe siècle. Néanmoins, dans les atlas, les flux sont davantage représentés sur les cartes représentant des données économiques à l'échelle du monde, où les transferts de café, thé et céréales (blé d'Argentine, des États-Unis ou du Canada vers l'Europe) croisent ceux - quelques pages plus loin - de la production mondiale industrielle (tissus, métallurgie, etc.) (Schrader et Gallouédec, 1923) (cf. Carte 2).

\footnotetext{
12 Un « premier groupe de flèches semble [...] illustrer des mobilités locales à courte distance inter-comtés [...]. Un second groupe semble représenter des flux internes, caractérisés par l'absence de franchissement de limite. Un troisième groupe de flèches est formé par des flux inter-états (entre I'Irlande et I'Angleterre) qui suggère des mobilités à plus longue distance "(Bahoken, $2013: 6)$.

13 L'année d'édition de la carte n'est pas précisée. Au regard de la vie de l'éditeur lliine Alekseï Alekseevitch (1832-1889), le document semble avoir été réalisé au cours de la deuxième moitié du XIXe siècle, voire peut-être à la même période que la carte de Ernst Georg Ravenstein (http://gallica.bnf.fr/ark:/12148/btv1b84451358.r=migration).

14 « 60 millions de candidats s'embarquèrent vers les Amériques entre 1820 et 1914 dans les pays de départ, le cas le plus inouï est celui de l'Irlande qui vit partir plus du tiers de sa population : 3 millions de départs entre 1845 et 1870 . Du côté des pays d'accueil, le rythme annuel des entrées aux États-Unis passe de 300000 vers 1850 à plus de 1 million à partir de 1900 et atteint même 1,4 million à la veille de la guerre " (Simon, 2008 : 32).

15 Voir également les travaux de Matthew Sankey sur le site Wikipédia qui lui est consacré (https://fr.wikipedia.org/wiki/Diagramme_de_Sankey).

16 La recherche, qui demande encore à être affinée, s'appuie sur des fouilles documentaires au sein de la Bibliothèque nationale de France (BNF), notamment à partir du site Gallica (http://gallica.bnf.fr), du département de géographie de I'Université de Poitiers et d'ouvrages respectifs des six auteurs de I'article.
} 


\section{Carte 1 : Carte figurative et approximative représentant} pour l'année 1858 les émigrants du globe

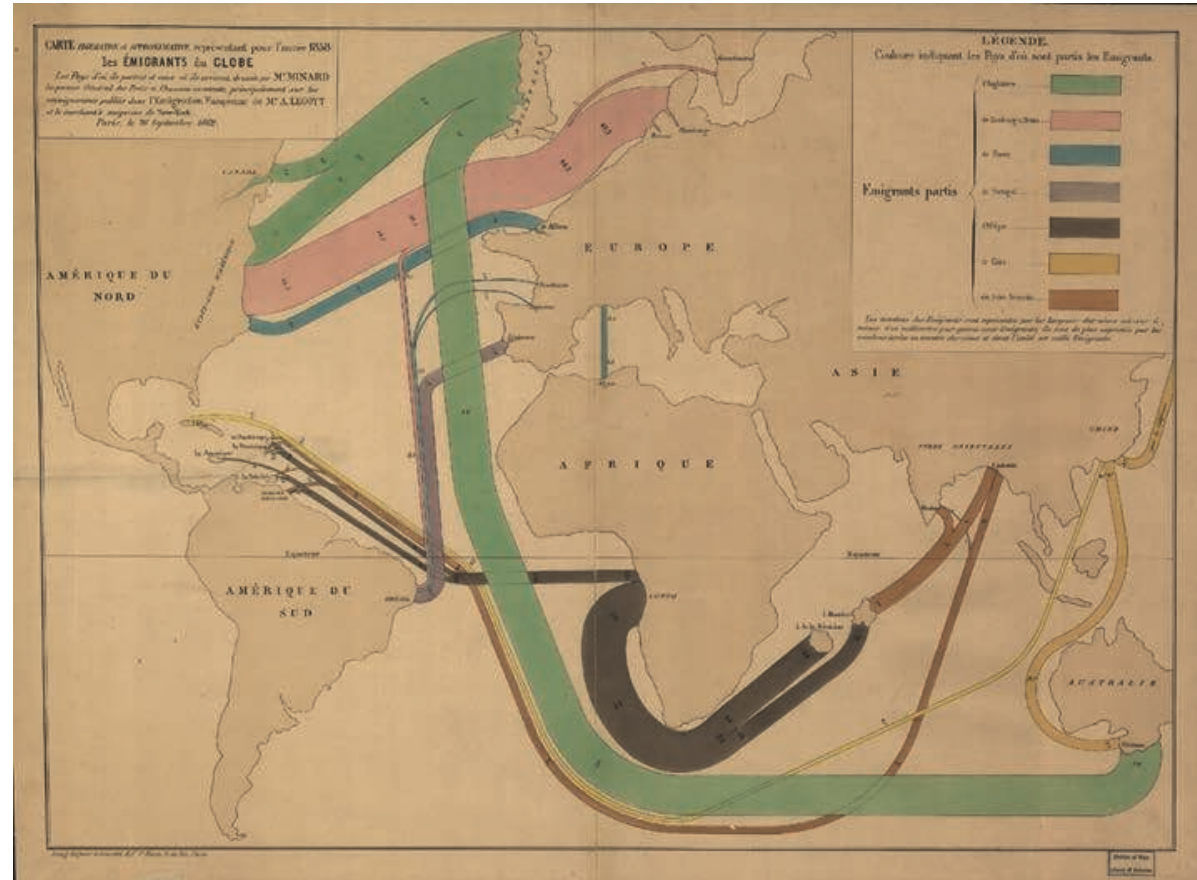

Source : https://cartographia.wordpress.com/category/charles-joseph-minard/

Crédit : Cartographia. Mapping The World Around You, Archive for the 'Charles Joseph Minard' Category, 2008.

Carte 2: Production mondiale des sols

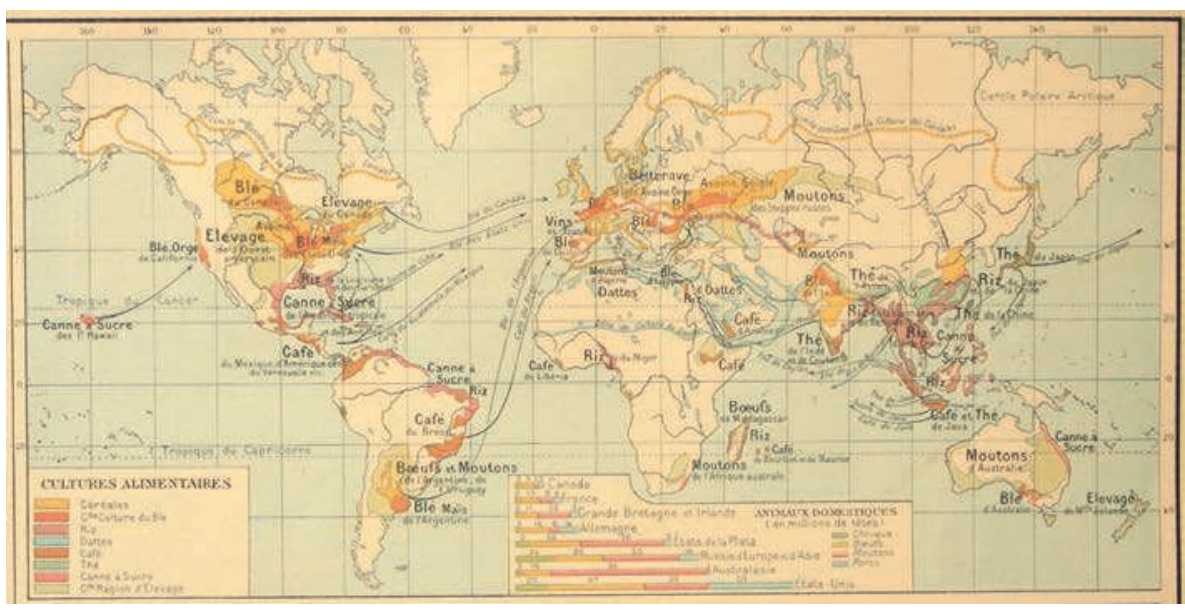

Source : Schrader Franz et Gallouédec Louis (1923) Atlas classique de géographie ancienne et moderne, Paris, Hachette, p. 123. 
L'absence de représentations des flux humains tient à des raisons essentiellement politiques : représenter les grandes migrations européennes aurait signifié mettre l'accent sur des sociétés "développées " en crise. Ainsi la structure spatiale des échanges économiques entre des couples de lieux d'origine (i) et de destination (j) a dominé l'objet des cartographies de flux jusque dans les années 1980, période à laquelle les études sur les migrations internationales contemporaines se développent.

\section{Des cartographies contemporaines de flux (sur)évaluant les processus migratoires}

Carte 3 : Les routes africaines de l'immigration clandestine

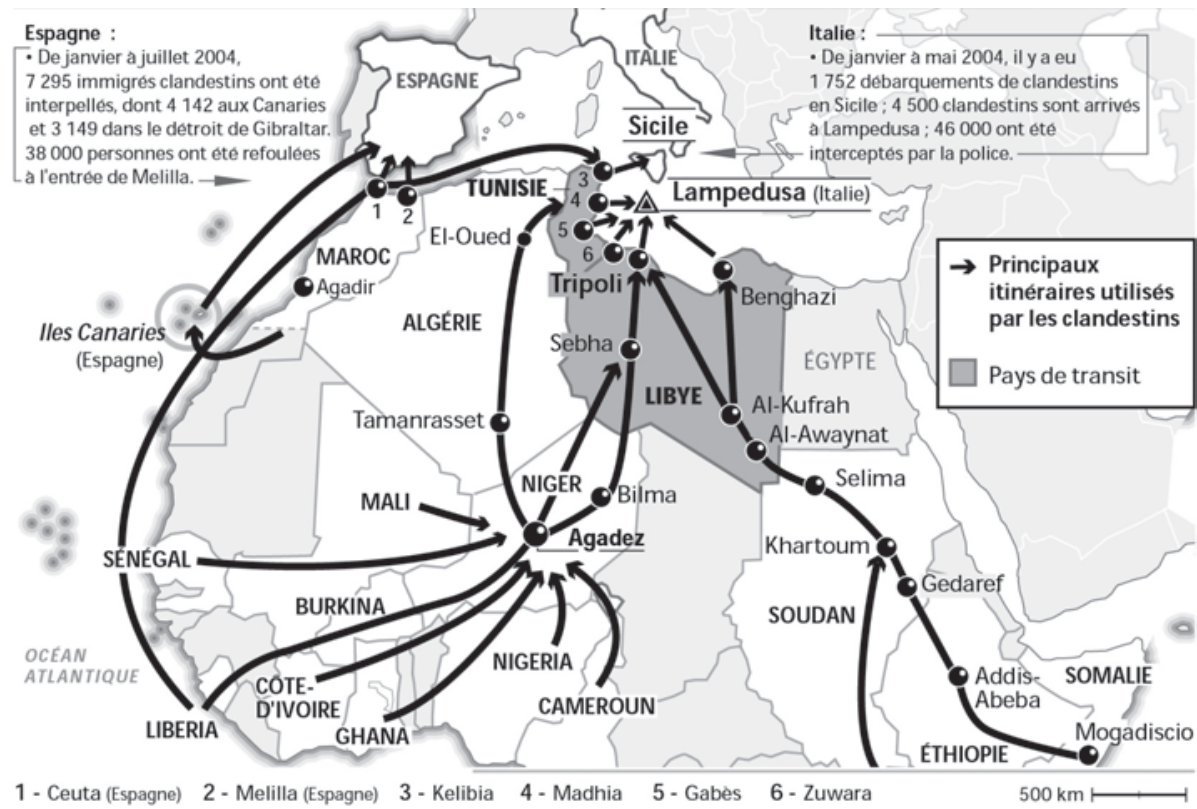

Source : http://www.lemonde.fr/international/infographie/2004/08/24/les-routesafricaines-de-I-immigration-clandestine_628348_3210.html

Crédit : Le Monde, 24 août 2004.

Certaines cartes publiées dans des revues scientifiques ou dans les médias laissent parfois penser que les déplacements de populations sont (très) importants statistiquement par rapport à la population mondiale. Ce type de représentation peut être lié aux couleurs utilisées : le noir ou le rouge foncé ne véhicule forcément pas le même message que des couleurs froides, comme le bleu ou le vert (Bertin, 1967). Des cartes élaborées par des "journaux de référence ", comme Le Monde et The Guardian, surinterprètent ainsi fréquemment les processus analysés (cf. Carte 3). À une période où les logiciels de cartographie étaient moins avancés, des cartes - comme celles publiées dans la REMI en 1992 - laissent a posteriori une impression d'arrivées massives, que la couleur noire - liée aux contraintes de publication de l'époque - renforce. Si pour Simone 
Donnefort ${ }^{17}$, " cette question ne se posait pas à l'époque ", pour Gildas Simon :

"L'objectif était surtout de montrer qu'il y avait une base d'informations derrière ce type de cartes. Par exemple, pour celles publiées en 1992, elles permettaient, pour l'une, de souligner l'importance des migrations, notamment turques et polonaises, vers l'Allemagne par rapport aux migrations allant du Maghreb vers la France ; pour l'autre, elle rappelait que l'immigration irlandaise vers le Royaume-Uni perdurait, etc. ${ }^{18}[. .$.$] À cette$ époque, nous étions très peu à réaliser ce type de cartes, chacun bricolait dans son coin, nous travaillions à tâtons et j'imagine que des cartes continuent de se faire ainsi. [...] Nous n'avions pas de recul sur ces graphismes qui à mon sens, étaient perçus différemment. Néanmoins plus le temps a passé, plus j'ai prêté de l'attention à ces représentations. Comme me l'a souvent rappelé Abel Bouillet qui m'a enseigné la cartographie, si la carte doit être lisible immédiatement, il est parfois important de ne pas trop ancrer les représentations dans l'imaginaire des lecteurs " (Entretien réalisé le 4 février 2016).

Représenter des mouvements de réfugiés, des circulations migratoires avec des flèches pourrait être décrit comme une "tentation cartographique ": I'image qu'elles procurent peut gêner aussi bien les cartographes que les lecteurs en donnant l'impression que les flèches dessinées sur la carte disent autre chose que ce que les cartographes auraient - parfois - souhaité transmettre. Les cartes représentant les flux de demandeurs d'asile vers l'Europe par exemple doivent être comparées avec la population européenne ou encore la population réfugiée aux abords des zones en conflit (près de $84 \%$ des réfugiés dans le monde en 2015). Si les flux apparaissent spectaculaires sur certaines cartes, la confrontation avec d'autres échelles doit permettre de les relativiser. Rappelons qu'entre 2014 et 2015, le nombre de demandeurs d'asile en Europe a augmenté, mais le total de ces migrants ne représente pas plus de $0,3 \%$ de la population de l'Union européenne aux mêmes dates. Ces représentations peuvent être liées à un " effet loupe ", à l'image de certains reportages de télévision montrant l'arrivée de personnes à une frontière sur un plan serré19. Ici, c'est le cartographe, qui, en élargissant le trait de la flèche, renforce l'illusion de flux importants.

Les cartes représentant les flux de la migration dite "clandestine " et dont les principaux éléments sont des flèches sans valeur proportionnelle ${ }^{20}$, conduisent, par ailleurs, à minimiser, voire invisibiliser d'autres migrations internationales dont les données statistiques sont disponibles. Par exemple, seule une partie des migrations clandestines transitant par l'espace sahélien se dirige ensuite vers le continent européen, les migrations régionales au sein de l'Afrique sont bien plus importantes (Lessault et Beauchemin, 2009). En gommant, volontairement ou non, la diversité des mouvements de populations dans la partie septentrionale de l'Afrique, les migrations se résument trop souvent aux routes empruntées par des réseaux mafieux. II ne s'agit pas de nier l'existence de ces économies criminelles dont les politiques migratoires européennes sont en partie responsables. La surreprésentation des liens entre immigration et crimi-

17 Diplômée de l'École supérieure de cartographie géographique en1967, elle fut cartographe à Migrinter depuis sa création jusque dans les années 1990. Elle était très proche de Michèle Béguin décédée le 12 mars 2014.

18 Cf. Simon, 1992.

19 Voir par exemple La nuit des réfugiés avec six reportages diffusés sur Arte le 6 octobre 2015.

20 Par définition, ces migrations ne peuvent évidemment pas être comptabilisées. 
nalité se renforce lorsque sur les cartes, les routes migratoires sont associées à celles des trafics d'armes, de drogues ou autres produits de contrebande. On peut citer la carte "Trans-Sahara Trafficking and Threat Finance " (cf. Carte 4) qui représente les trafics illicites vers la Libye depuis le Mali, I'Algérie, le Tchad, le Soudan et l'Égypte. Cette carte propose des interprétations erronées dans le sens où les réponses à la lutte contre le crime organisé (armes, drogues) ne peuvent pas être les mêmes que celles qui peuvent être apportées à la migration des personnes en quête de protection ou d'une vie meilleure. Ces " inepties cartographiques " sont bien souvent utilisées dans la propagande des mouvements d'extrême droite xénophobes et opposés aux migrations. Par exemple, pour le Mouvement pour la Remigration, au regard de la carte 4 reproduite dans un de leurs rapports, "la fabrication de réfugiés [ne serait] qu'un secteur de la criminalité comme les autres [...]. Les groupes armés ["recruteraient"] ainsi toujours plus de réfugiés. Ces "migrants" [deviendraient] à leur insu les banquiers du crime et du terrorisme, finançant du matériel, des hommes, et des armes [...]. [Selon ce mouvement, il serait] crucial de réaffirmer l'ancrage des réfugiés sur leur propre territoire pour ne pas céder de terrain aux terroristes ${ }^{21}$.

\section{Carte 4 : Trans-Sahara Trafficking and Threat Finance, 22 April 2015}

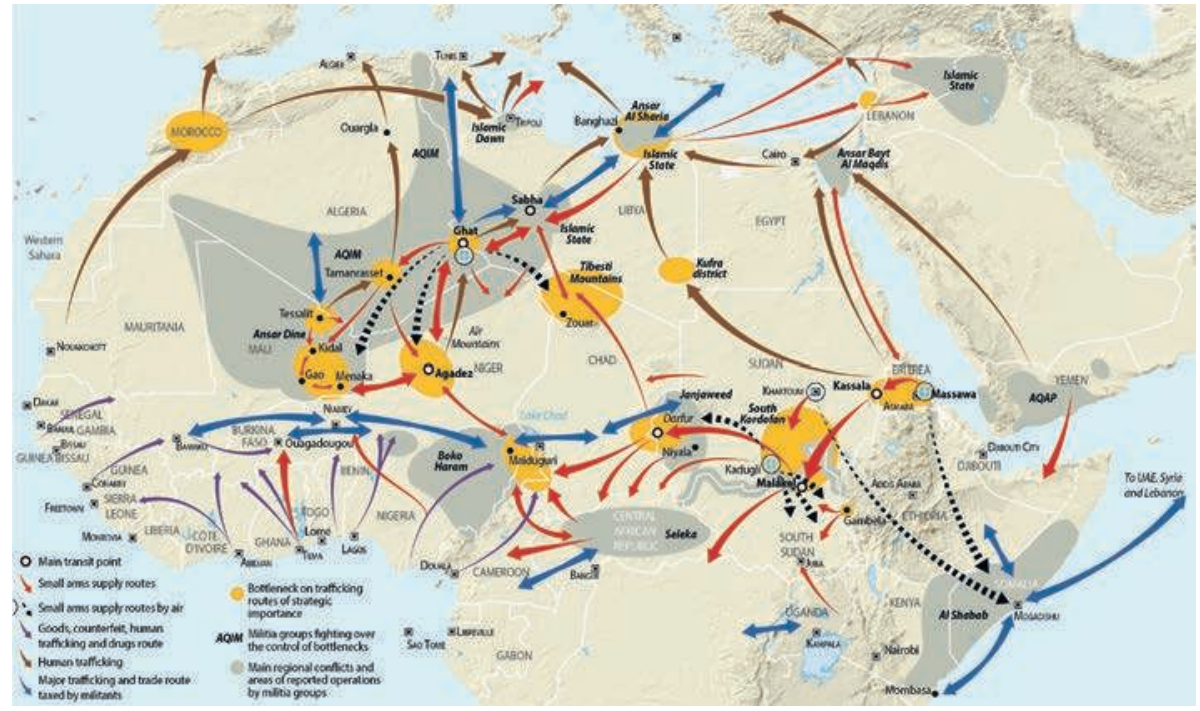

Source : Norwegian Center For Global Analysis, 2015.

Devant de tels propos, aussi faux qu'injustifiables juridiquement et politiquement, les cartographes se doivent de réfléchir à ce qu'ils produisent, et à la manière dont ils conçoivent les cartes. Une des questions est notamment de savoir s'ils doivent choisir entre " la flèche "-avec ou sans valeurs proportionnelles -, ou "l'itinéraire " pour essayer de tracer plus précisément le paysage de la migration en relation avec les contextes locaux et régionaux.

21 Mouvement pour la Remigration (2015) Rapport choc : pourquoi faut-il cesser de recevoir des migrants ?, [en ligne]. URL : http://www.mouvementpourlaremigration.fr/ blog/ (le site originel n'est plus disponible). Nous ne partageons pas les points de vue défendus par les auteurs de cet article. 


\section{Prise en compte et ignorance des contextes (géo)politiques}

Les représentations cartographiques des flux migratoires ignorent souvent les contraintes de la situation géopolitique régionale auxquelles les personnes sont confrontées. Par exemple, pour les réfugiés syriens fuyant la guerre depuis 2011, l'impossibilité de se déplacer vers Israël, les craintes des populations de confession sunnite à aller vers l'Irak et I'Iran, les difficultés pour rejoindre les États du Golfe et du Caucase du Sud, ne sont pas indiquées sur les cartes. Ces différents éléments expliquent pourtant en partie que les réfugiés soient situés majoritairement en Jordanie, au Liban et en Turquie et que certains s'orientent ensuite vers des pays d'Afrique, d'Europe voire d'Amérique latine. La majorité des cartes de flux migratoires parviennent en effet à la perception d'un territoire où les difficultés sociales, les obstacles administratifs, (géo)politiques et la distance ne sont pas pris en compte. "Les concepteurs de ces cartes [dont nous faisons parfois partie] opèrent ainsi de nombreux raccourcis qu'ils imposent au lecteur ; ils laissent de côté les questions essentielles, mais peu documentées de la hiérarchisation des flux ou de l'importance de telle ou telle agglomération le long de ces routes, ou encore de la variabilité du phénomène, de sa saisonnalité [...] " (Choplin et Pliez, 2011).

Il est difficile de figurer toutes ces informations sur une même carte au risque d'avoir un document difficilement compréhensible. Néanmoins, représenter des éléments qui expliquent en partie l'environnement dans lequel les migrations des personnes s'effectuent est important. Ce type de cartographie dessine diverses "rugosités " à travers lesquelles les parcours s'élaborent. En regardant par exemple la carte 5 intitulée " Expérience migratoire et justice spatiale. L'itinéraire de Rokyata, mineure ivoirienne, du Sahel aux rives de la Méditerranée ", issue des travaux de Nelly Robin (2014: 104), on constate que ces jeunes migrants - au-delà du fait qu'ils ont recours à différents moyens de transport (maritime, terrestre et aérien) - ont des parcours qui se déploient différemment selon les étapes. À certains moments, les personnes peuvent voyager de manière autonome, à d'autres, elles ont recours à des moyens illicites ou sont prises dans des réseaux de traite. Ces diverses ressources, plus ou moins choisies, parfois complètement subies, se retrouvent aussi bien dans la circulation que dans l'attente au sein des villes ou des espaces frontaliers ou au sein de leurs activités économiques. L'objectif de ce type de carte qualitative est de saisir les itinéraires composant les flux migratoires " dans leur diversité et leur richesse, de les englober et de les unifier tout en tenant compte des leurs multiples formes, mécanismes et pratiques" (Robin, 2014). Au sein de ces parcours, nous savons également que les migrants peuvent être soumis à divers statuts juridiques selon le pays où ils se trouvent. Ils peuvent aussi être confrontés à des contrôles et/ou bénéficier de mesures de protection conduisant à vivre des situations très hétérogènes selon les pays (Migreurop, 2012 : 121-123), dessinant ainsi une "territorialité du risque juridique " (Robin, 2014 : 113). 
Carte 5 : Expérience migratoire et justice spatiale. L'itinéraire de Rokyata, mineure ivoirienne, du Sahel aux rives de la Méditerranée

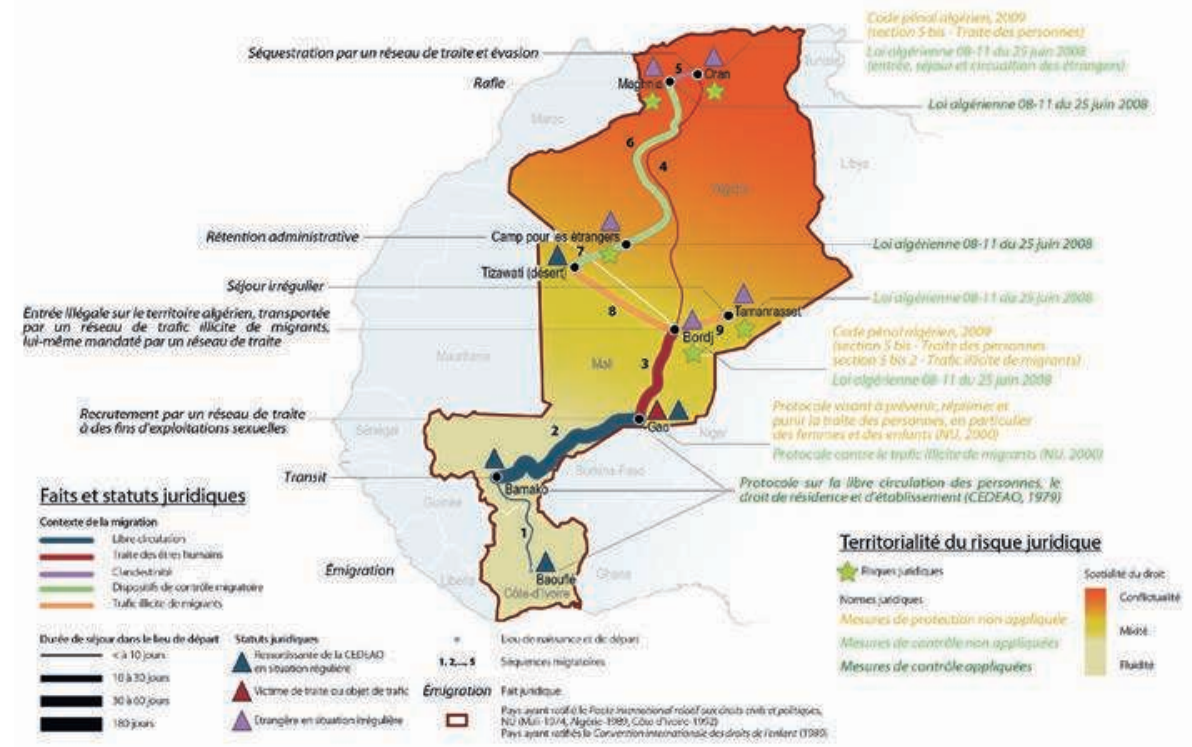

Source : Robin Nelly (2013) Enquête " Mineurs migrants en transit ".

Crédit : Migrinter, 2013 - Pôle carto.

Dans la plupart des cartes de flux migratoires, les auteurs adoptent une perspective surplombante qui évince le point de vue des personnes migrantes. Pourtant, la représentation de données quantitatives agrégées n'est pas nécessairement opposée à des données qualitatives et explicatives. Ainsi, Florence Boyer (2005: 432) a montré que les Nigériens effectuant des migrations circulaires entre Bankilaré (Niger) et Abidjan (Côte d'Ivoire) via Niamey (Niger), qualifiaient différemment les étapes de leur parcours migratoire : certains tronçons s'apparentent à des "lieux de recréation de l'intimité sociale ", d'autres, connus par l'histoire migratoire, sont relativement rassurants, alors que ceux qui leur sont étrangers sont assimilés au danger (cf. Carte 6).

L'auteure précise par ailleurs que " les migrations circulaires [et autres mobilités internationales] ne sont pas géographiquement situées, au sens de localisées sur une carte, mais elles mettent en jeu une pluralité de localisations, de même qu'elles mettent en jeu le mouvement "(Boyer, 2005). Pour le dire autrement, il ne faut pas oublier que les parcours migratoires sont liés à des contextes politiques locaux, régionaux et continentaux qui agissent comme des ressorts dans la dynamique des migrations. Montrer au sein de ces "pièces de théâtre cartographiques ", les " décors " et les " acteurs " ${ }^{22}$ (Rekacewicz, 2014),

22 Philippe Rekacewicz, Cartes en colère, exposition de cartes à la Maison des métallos, Paris, octobre 2012. 
et qui ne cessent de se recomposer au fil des étapes, permet de rappeler l'importance des contextes (géo)politiques.

Carte 6 : Les exodants : qualifier les lieux au fil du mouvement

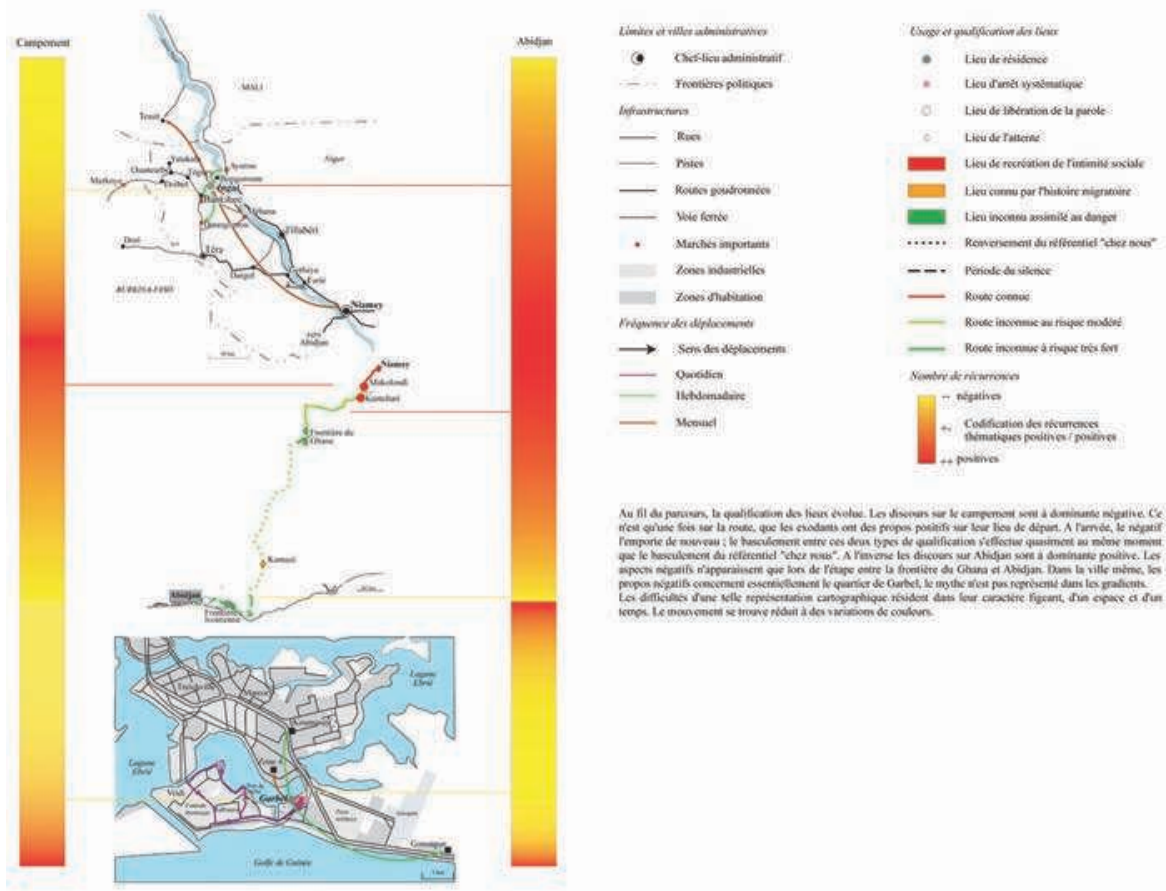

Source : Boyer Florence (2001) Entretiens, observations, Ingui, route Niamey/Abidjan, Abidjan, mai-nov. 2001.

Crédit : Migrinter, 2005.

\section{Représenter les flux migratoires : un défi cartographique qui perdure}

En 2015, 197 pays étaient reconnus par les Nations unies. Une carte où serait représentée l'intégralité des flux entre les différents États devrait faire figurer près de 38000 flèches matérialisant le lien de chaque pays avec les autres pays du monde ${ }^{23}$. Une telle carte serait illisible. Par ailleurs, les circulations sont très complexes. Dans la nomenclature migratoire, beaucoup de pays remplissent plusieurs fonctions: I'Espagne et l'Ukraine, par exemple, sont autant des pays de départ que de transit et d'arrivée. Les typologies sont difficiles à établir. Or, la mise en ordre de l'espace géographique ou la manière dont les cartographes représentent le territoire, est l'une des conditions pour rendre pensable l'espace du monde représenté (Jacob, 1992). Il faut donc faire des choix. 


\section{L'articulation entre les données et la sémiologie graphique}

La cartographie des migrations peut s'appréhender à travers différents types de données: des données qualitatives (récits de parcours), des données quantitatives absolues, brutes (variable de stocks et matrices origine/destination) et des données quantitatives relatives, transformées (des indicateurs). Au regard des règles de la sémiologie graphique, théorisées et formalisées par Bertin (1967), chaque type de données détermine des modes de représentations distincts : des classes de valeurs pour les données relatives, des symboles proportionnels pour des données de stocks et des flèches de tailles proportionnelles pour des données de flux quantitatifs.

Très diversifiée et hétérogène, la production cartographique sur les migrations de population semble présenter deux tendances opposées.

Tout d'abord, on peut noter que les cartes contribuent souvent à une " immobilisation " des processus décrits, en privilégiant l'utilisation de données statiques, en particulier des variables de stocks. Ce choix favorise les cartographies en symboles proportionnels et montre les migrations sous une forme d'ancrage (c'est-à-dire des pays d'où viennent les migrants ou ceux dans lesquels ils arrivent/ou restent temporairement, dans un temps plus ou moins long). La représentation du mouvement y est détournée en mettant en évidence un état de la résultante d'un phénomène, plutôt que le phénomène lui-même (Bahoken, 2009a). Plus faciles à fabriquer, ces cartes résolvent de fait la question de la sélection des flux pertinents.

À l'inverse, les nouvelles technologies (outils de conception graphique, interactivité, animation, etc.) conduisent à " dynamiser " les cartes représentant les migrations. Ce processus peut se lire, d'une part dans la production de flèches proportionnelles, choix sémiologique relativement difficile à concevoir et à dessiner (superposition, courbures des flèches, détermination des tracés qui font sens, sinuosités, taille proportionnelle aux autres éléments de la carte), mais parfois utile pour comprendre certains enjeux de circulations et l'importance de certains déplacements. D'autre part, la carte peut s'appuyer sur la production de flèches qualitatives à partir d'informations éparses, voire incomplètes, pouvant conduire à des messages cartographiques erronés.

\section{Échelles et temporalités}

Un des enjeux de ces cartographies semble se jouer dans la complexité et la prise en main des informations disponibles. En effet, les phénomènes migratoires présentent une dimension sociale (type de population), une dimension spatiale complexe (origine, destination, étapes, retours, etc.) et une dimension temporelle forte (mouvement des déplacements, temps d'attente, évolution des phénomènes), particulièrement difficiles à articuler pour les cartographes, les obligeant à penser leur carte en " plusieurs niveaux de lecture ".

Afin de combiner ces différents aspects au sein d'un document cartographique, il est nécessaire de réaliser des opérations à la fois sur les données et sur les modes de représentation. Les facettes des processus migratoires apparaissent différemment selon les échelles d'analyse et les niveaux d'agrégations choisis. Des sélections d'ordre statistique peuvent par exemple permettre d'es- 
tomper un effet " spaghetti " lors d'une superposition importante de flux. D'un point de vue géographique, changer d'échelle ou de niveau d'agrégation permet de passer d'une image globale à une échelle plus locale, jusqu'à abandonner les représentations agrégées pour narrer les histoires individuelles.

En effet, la thématique migratoire propose un retour à la cartographie des itinéraires et des parcours. Mais contrairement à la dimension descriptive des illustrations des itinéraires de voyages et d'exploration, cette cartographie se veut analytique en tentant de saisir les contraintes subies par celles et ceux qui effectuent le parcours et qui n'apparaissent pas dans les données statistiques.

Plutôt qu'une carte globale, il s'agit alors de représenter l'ensemble des lieux de l'itinéraire d'une personne. Dans cette démarche, la représentation de la dimension temporelle permet de saisir des contraintes qui n'apparaissent pas au niveau spatial (attente, errance). Mais à cette complexité méthodologique s'ajoute alors une relative complexité technologique (animation, 3D) qui peut représenter un frein (coût, temps de traitement) pour la production cartographique.

\section{Nouvelles technologies, nouvelles cartographies?}

"Avec l'ordinateur, on peut faire bouger les cartes " (Brunet, 1987). On peut les animer et rendre possible l'interaction avec I'utilisateur. Dans le cadre de la cartographie des flux migratoires, ce dernier point est un moyen souvent utilisé pour transférer la responsabilité de la sélection de l'information du cartographe à l'usager de la carte. Ainsi, de nombreux sites proposent des cartes dans lesquelles il est possible de cliquer sur tel ou tel pays pour voir se dessiner les flux de populations entrantes ou sortantes. Ces mouvements se matérialisent tantôt par des symboles proportionnels ${ }^{24}$, des gradations de couleurs ou des lignes plus ou moins épaisses ${ }^{25}$. Certaines représentations inspirées de l'infographie peuvent être aussi relativement originales ${ }^{26}$. Combinées avec l'animation temporelle, ces cartes permettent de tracer l'évolution dans le temps des flux migratoires pays par pays. L'exposition de Raymond Depardon et Paul Virilio, "Terre natale. Ailleurs commence ici ", scénographiée par les artistes et architectes américains Diller Scofidio, Renfro et Mark Hansen, Laura Kurgan et Ben Rubin, offre, dans une salle de près de 400 mètres carrés, des visualisations à 360 degrés : celle des migrations de réfugiés au cours des cinq dernières décennies, et celle des transferts d'argent des migrants. Elles font sûrement partie des réalisations les plus réussies à ce jour ${ }^{27}$.

Néanmoins, lorsque les initiateurs de ces projets de cartographie dynamique laissent le choix aux usagers de sélectionner eux-mêmes l'information (indicateur, année, pays), les cartographes, ainsi transformés en développeurs, ne jouent plus leur rôle de thématiciens puisqu'ils renoncent à livrer des clefs de lecture globale (ou du moins son intention, sa vision du monde). Dans ce type

\footnotetext{
$24 \mathrm{http}: / / w w w . t h e r e f u g e e p r o j e c t . o r g$

25 http://migrationsmap.net/

26 https://www.iom.int/world-migration

27 Elle a été présentée pour la première fois à Paris, du 21 novembre 2008 au 15 mars 2009, à la Fondation Cartier pour I'art contemporain.
} 
d'application, la carte n'est plus un moyen de matérialiser et donner à voir un regard spécifique sur le monde, elle est construite par le développeur de l'application et par son utilisateur qui va générer, à la demande, des représentations spécifiques.

Mais ces nouvelles applications cartographiques dynamiques ne sont pas dégagées des enjeux politiques et éthiques : on peut parfois y déceler - comme pour la cartographie thématique classique ou conventionnelle - des intentions politiques. Le 26 octobre 2015, I'agence d'informations finlandaise Lucify ${ }^{28}$ publiait une carte interactive (cf. Carte 7) et animée permettant de visualiser les flux de demandeurs d'asile arrivant en Europe entre janvier 2012 et septembre 2015. Repérée par The Independent (27 octobre 2015) et diffusée par la presse française (Slate, le 29 octobre 2015, l'Observateur, le 30 octobre 2015), cette carte s'est rependue sur Internet comme une traînée de poudre. Tout d'abord, sur cette carte européocentrique, seuls les flux vers les pays " européens " sont représentés. Les réfugiés accueillis dans les autres pays (tels la Jordanie, le Liban et la Turquie qui en accueillent pourtant la majorité) ne sont pas représentés. Deuxièmement, les choix graphiques utilisés pour représenter l'information sont également significatifs des intentions des auteurs. Sur cette carte animée, les mouvements des demandeurs d'asile sont représentés par de petits traits qui se déplacent des pays de départ vers les pays d'arrivée. Contrairement à ce qui peut sembler au premier abord, cette carte ne se situe donc pas au niveau des parcours individuels. Elle ne raconte pas les histoires des migrants. Loin des réalités sinueuses des parcours migratoires réels, ici, chaque trait (qui représente vingt-cinq ou cinquante personnes) suit une trajectoire rectiligne comme un missile lancé vers l'Europe. Au final, cette sémiologie graphique animée met en scène un scénario d'invasion quasi militaire, avec des pays européens attaqués - et envahis - par les étrangers. II est difficile de savoir si la rhétorique d'invasion de cette carte est consciente et volontairement choisie. Ce qui est certain, c'est que cette agence non spécialisée en cartographie voulait montrer " I'ampleur de la crise " des réfugiés. Cette carte résulte probablement à la fois d'une maladresse (la sélection des données) et de la volonté de retranscrire graphiquement une idée préconçue (I'arrivée massive d'exilés en l'Europe).

Au-delà de la sémiologie graphique, une carte résulte d'un processus technique et intellectuel qui consiste à convertir des données concrètes plus ou moins connues en une représentation abstraite (Lacoste, 1976). Or, cette abstraction graphique remplace la richesse contenue dans la multiplicité des histoires vécues par une représentation holiste qui tente de porter un regard sur

\footnotetext{
28 Lucify est une agence de data visualisation basée à Helsinski. Lucify, qui signifie " rendre lucide ou clair " en anglais, se propose "d'aider " les clients à visualiser leurs données sous forme de représentation interactives, pas forcément cartographiques. Les visualisations proposées, développées pour le web, sont facilement intégrables sur un site ou un blog et les codes sources de leurs visualisations sont parfois diffusés librement. Selon le site internet de I'agence, un certain nombre de réalisations ont été intégrées dans plusieurs journaux en ligne: The Guardian, The Atlantic, Huffington Post, The Independent, Helsingin Sanomat et Daily Mail. Les deux auteurs de la carte sont cofondateurs de l'agence. Ville Saarinen travaille dans la communication et le développement de logiciels. Selon la description qu'il donne sur le site de l'agence, il passe beaucoup de temps à penser la combinaison de la sociologie et de la technologie. Juho Ojala se présente quant à lui comme fasciné par la communication et curieux d'idées dangereuses. L'agence a produit d'autres infographies sur le même sujet (http://www. lucify.com/).
} 
le monde. De fait, les cartes de géographie, leur construction, leur sémiologie passent bien souvent à côté de la réalité. Elles dessinent et schématisent la géographie des phénomènes spatiaux, mais les racontent mal. Les cartes des migrants morts aux frontières ${ }^{29}$ permettent-elles de rendre compte des drames humains qui ne cessent de se succéder ? Et que veut dire "rendre compte " de ces drames depuis la cartographie et plus largement la science ? Si la sémiologie graphique utilisée dans la cartographie scientifique ne peut pas tout, qu'en est-il de l'approche artistique ? L'art permet-il de raconter autrement l'espace géographique?

Carte 7 : The Flow towards Europe, Lucify, 26 octobre 2015

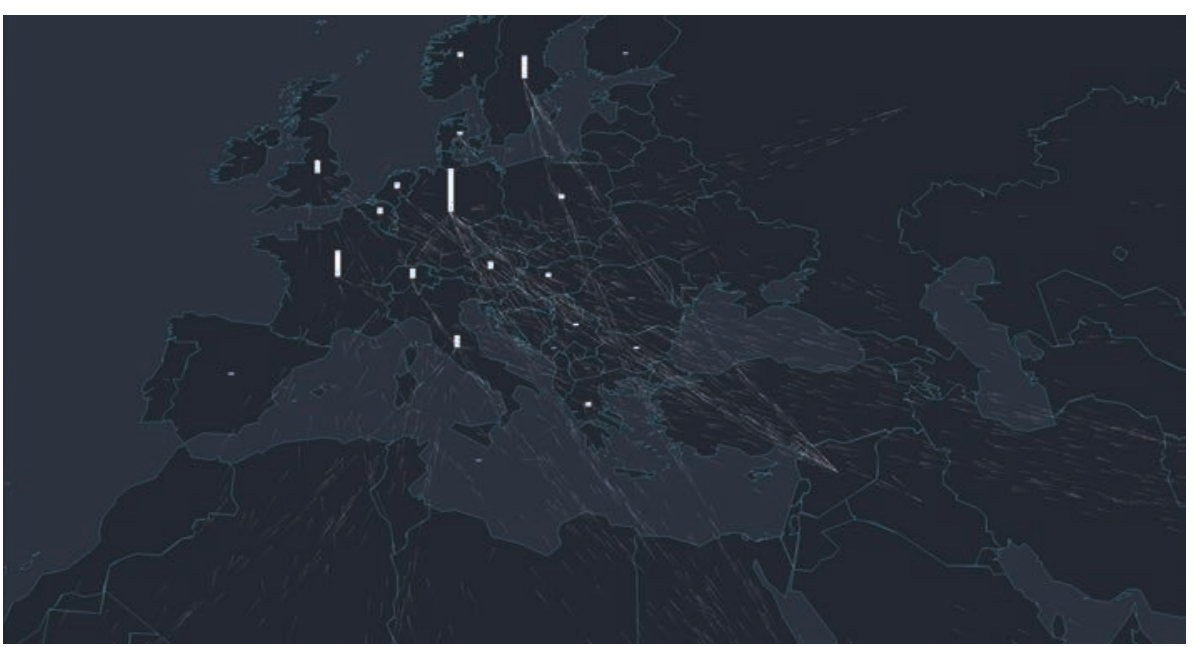

Source : http://www.lucify.com/the-flow-towards-europe/ Crédit : Ville Saarinen et Juho Ojala, 2015 ; MIT License (MIT) https://github.com/lucified/lucify-refugees

\section{Cartographies des migrations entre art, science, politique et militantisme}

Parmi les cartographies ayant pour objet les migrations internationales, certaines réalisations se distinguent des représentations conventionnelles, regroupées dans les manuels scolaires, les ouvrages universitaires ou les atlas. Ces cartographies défient les règles des fonds de carte institutionnalisés et de la sémiologie graphique. Elles inventent des langages nouveaux et bouleversent nos repères habituels de fabrication et de lecture de cartes. Ces productions, individuelles ou collectives, sont le fait de cartographes, d'artistes, de militant.e.s, de personnes en situation d'exil. Si elles constituent un corpus très hétérogène, elles relèvent toutes, à différents degrés, d'un positionnement que l'on pourrait qualifier de " critique ", d'" expérimental " (Crampton et Krygier, 2005 ; Gintrac,

29 Lambert Nicolas (2015) Mer Morte (1993-2015), Carnet (néo)cartographique, [en ligne]. URL : http://f.hypotheses.org/wp-content/blogs.dir/1381/files/2015/04/MerMorte.png 
2012), d' " indisciplinaire " ou encore de " participatif " (Mitchell, 1995), chacun de ces termes renvoyant à des horizons épistémologiques distincts. En interrogeant les normes de représentation, ces cartes visent à défier le projet positiviste de neutralité du savoir. Sans prétendre à l'exhaustivité, nous proposons dans cette troisième partie, d'explorer la nébuleuse de ces cartographies migratoires critiques, en tentant de dégager au moins deux tendances principales : celles qui donnent à voir, selon la métaphore du dévoilement, les dispositifs de contrôle et de surveillance des migrations afin de les dénoncer; et celles qui documentent les parcours et les expériences migratoires individuelles et collectives. Ces deux catégories n'épuisent évidemment pas la richesse des perspectives adoptées. Amenée à être complétée et discutée, cette typologie ouverte est pensée comme une invitation à poursuivre la réflexion sur les modes de figuration des processus migratoires.

\section{Cartographies de " dévoilement " des dispositifs du contrôle migratoire}

Les réalisations qui suivent prennent le contre-pied des cartographies migratoires de flux : elles ne représentent pas le mouvement, mais tout ce qui le contrarie, le restreint, le prolonge ou le réoriente. Par la mise en visibilité des dispositifs de contrôle, ces cartes servent d'arguments scientifiques et politiques, voire parfois de preuves juridiques.

L'Atlas des migrants en Europe, sous-titré Géographie critique des politiques migratoires (Migreurop, 2012) (30 $^{30}$ produit par le collectif scientifique et militant Migreurop, s'inscrit dans cette "topique de la dénonciation " (Boltanski, 2007 : 113). Sur fond de carte et à l'appui d'une sémiologie graphique conventionnelle, I'apport critique tient ici aux données représentées ${ }^{31}$. Si de nombreuses cartes de I'Atlas des migrants en Europe utilisent des données compilées par des organismes chargés de comptabiliser afin de surveiller les migrations ${ }^{32}$, ces données sont en partie subverties pour faire la démonstration des conséquences néfastes, voire meurtrières des dispositifs de contrôle.

$\mathrm{D}^{\prime}$ autres réalisations opèrent une critique des politiques migratoires en représentant des données permettant de comprendre leurs fonctionnements et leurs effets, mais aussi en bouleversant les conventions cartographiques.

Dans son blog Carnet Néocartographique, Nicolas Lambert, un des auteurs de l'Atlas des migrants en Europe, présente des " métaphores cartogra-

30 Une première version était parue en 2009.

31 À titre d'exemple, la carte des lieux de détention arbitraire des étrangers au Maroc, imaginée et réalisée en février 2015 par une des membres du collectif, Elsa Tyszler, est symptomatique de l'enjeu cartographique. Pour la première fois, une carte montrait a minima le nombre d'étrangers détenus dans dix-huit villes du Maroc (écoles, centres sociaux, complexes sportifs, etc.). Elle a non seulement eu un impact dans différents médias, mais elle a également conduit le président du tribunal administratif de Casablanca à dépêcher un huissier sur plusieurs lieux, afin d'ouvrir une enquête suite à la grande rafle menée par les autorités marocaines dans les camps établis proche de I'enclave espagnole de Melilla.

32 Voir par exemple les cartes des personnes soumises aux apories administratives de la Convention de Dublin, réalisées à partir de données produites par Eurostat (Migreurop, 2012 : 60-63). 
phiques ${ }^{33}$ créées avec Olivier Clochard (Clochard et Lambert, 2015). À partir de statistiques sur la mortalité migratoire aux frontières de I'Union européenne et de dessins de presse, trois cartes explorent des figurations frontalières inédites. La combinaison des sources statistiques et médiatiques dans l'élaboration de ces cartes donne lieu à des travaux qui permettent de s'interroger autant sur les processus migratoires (ici, à partir du taux de mortalité en migration) que sur leur médiatisation.

La Cartographie critique du détroit de Gibraltar (2004), réalisée par le collectif d'artistes, scientifiques et militant.e.s Hackitectura ${ }^{34}$, basé en Espagne, fait office de " carte tactique " (cf. Carte 8). Au fond de carte référentiel sur le détroit de Gibraltar, sont associées des données sur les localisations et le fonctionnement des dispositifs de surveillance de I'Union européenne. La représentation de la terre en jaune, de la mer en noir et le foisonnement des symboles de dispositifs de surveillance servent un propos critique sur l'hypersécurisation et la militarisation de cet espace.

Dans un registre où le référentiel topographique est absent, Migmap, programme de création artistique, produit par la plateforme de conservation et d'exposition d'œuvre d'art k3000 établie en Allemagne, est une création en quatre volets de visualisation des politiques migratoires européennes ${ }^{35}$. Sont ici cartographiés, à partir de figurations dynamiques et interactives, les discours politiques, les réseaux d'acteurs, les lieux et pratiques significatifs des politiques migratoires, ainsi que le processus d'européanisation dont elles émanent (cf. Carte 9).

Enfin, au-delà de cartes mises au service de démonstrations scientifiques et politiques, le travail du collectif d'artistes, d'activistes, de chercheur.se.s Forensic Architecture, investit la carte en tant que preuve juridique. À partir de sources diverses, et notamment d'images provenant des dispositifs de surveillance en Méditerranée, le projet The Let-to-Die Boat (Heller et Pezzani, 2014) reconstitue la cartographie du naufrage d'un navire et de ses passagers en exil. Ce document a servi de preuve dans le cadre d'un procès attenté par une coalition d'ONG contre plusieurs États membres de I'OTAN, accusés de non-assistance aux naufragé.e.s, et a été utilisé en tant que modèle de preuve dans d'autres procès du même type.

À cette entreprise de visibilisation des effets des politiques migratoires, répond une autre forme de critique qui entend documenter les expériences individuelles et collectives.

33 Les cartes qui sont visibles sur le site (néo)cartographie (http://neocarto.hypotheses. org/1731) ont été également publiées dans I'ouvrage coordonné par Amilhat Szary et Giraut (Clochard et Lambert, 2015).

34 Hackitectura.net (2001-2012) est un collectif d'architectes, de programmeurs, d'artistes et d'activistes (Pablo Soto, Sergio Moreno, Jose Perez de Lama, etc.) qui se consacrent à l'étude des corps en mouvement et des flux électroniques, dans des territoires émergents. Voir également leurs autres projets : GISS (Stream Global Support indépendant, 2005-2007), Indymedia Détroit (2003-2007), Fadaiat (Tarifa-Tanger, 2004-2005), Emerging Géographies TCS2, Estrémadure (2007) ou Libertés Plaza, Sevilla (2005-2007).

35 k3000 inclut Helmut Dietrich, Matthew Gaskins, Sophie Goltz, Nana Heidenreich, Sabine Hess, Sylvia Kafhesy, Serhat Karakayali, Astrid Kusser, Maureen Müller, Efthimia Panagiotidis, Susanna Perin, Peter Spillmann, Vassilis Tsianos, Michael Vögeli et Marion von Osten. 


\section{Carte 8 : Extrait d'une partie de la carte réalisée par Hackitectura}

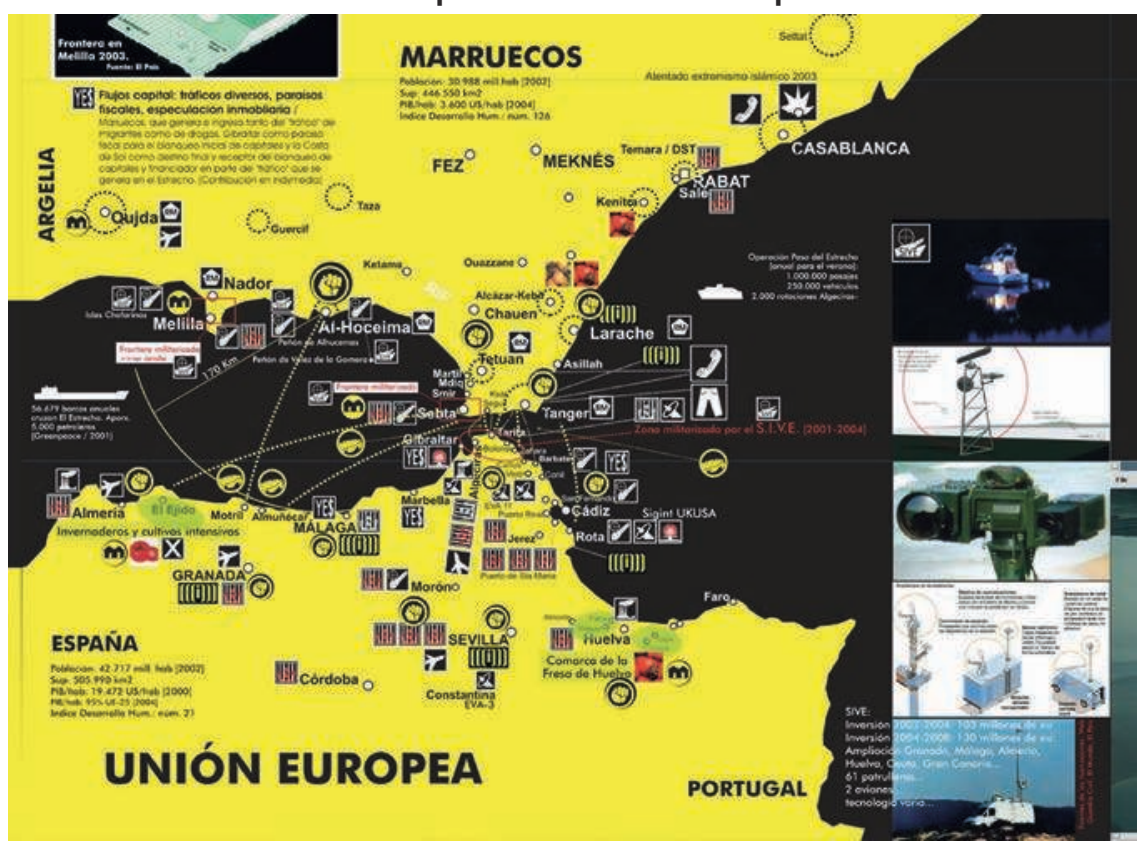

Source : http://x.hackitectura.net

Crédit : Hackitectura.net, 2017.

\section{Carte 9 : Discourses}

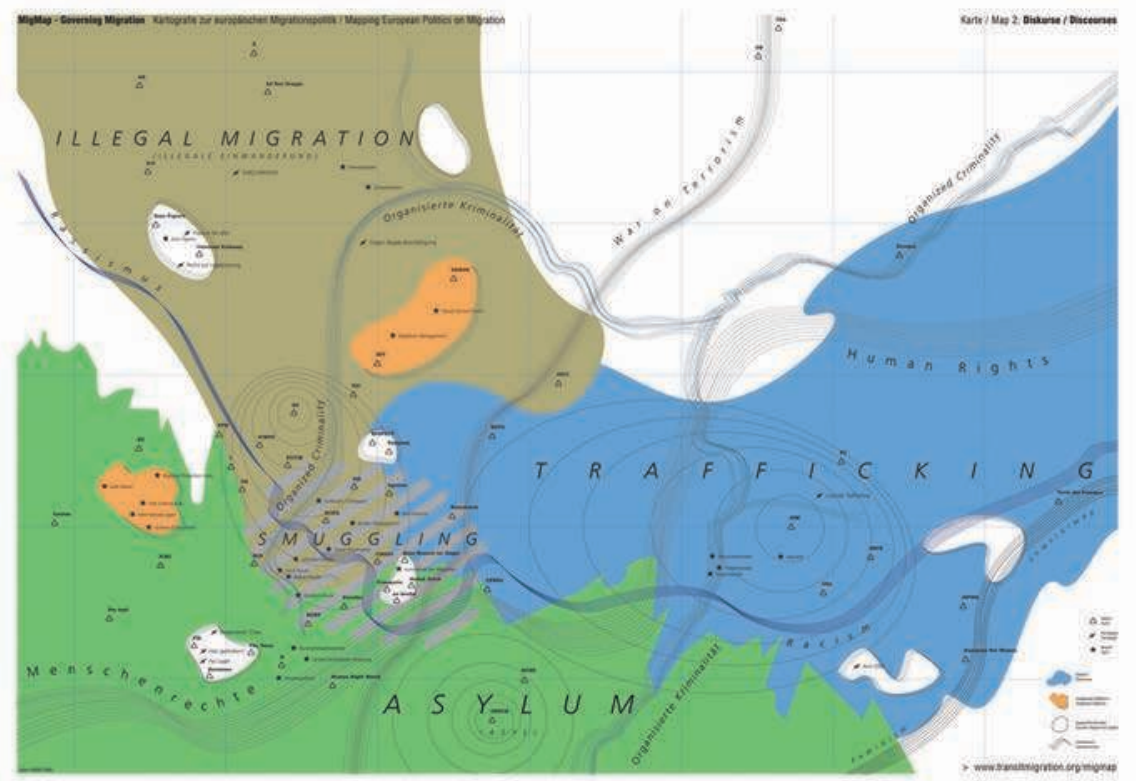

Source : http://www.transitmigration.org/migmap/home_map2.html

Crédit : Labor k3000, 2004. 
Deux projets se situent à l'interface des cartes de " dévoilement " des politiques migratoires et des cartes d'expériences vécues par les individus et les groupes. On peut citer dans ce registre la cartographie participative et dynamique Close the Camps, créée par Migreurop en 2013, qui cherche à faire connaitre I'Europe des "camps " en " mobilis[ant] toutes celles et ceux qui s'opposent aux mécanismes d'enfermement et d'éloignement des migrants en vue de défendre leurs droits fondamentaux ". Des éléments d'expériences de personnes ayant fait l'expérience des camps d'étrangers sont croisés à un projet de recensement de ces camps. On peut également citer une série de cartes réalisées par Philippe Rekacewicz et publiées à la fois dans Le Monde diplomatique et sur le blog Visionscarto. Dessinées à la main, elles rompent avec I'illusion de "cartes objectives " et sans auteur, et réhabilitent la sensibilité des cartographes. La singularité de ce geste cartographique, en esquisse, humanise la carte, et au-delà de la carte, le sujet qu'elle représente.

Carte 10 : La « Grande roue africaine »

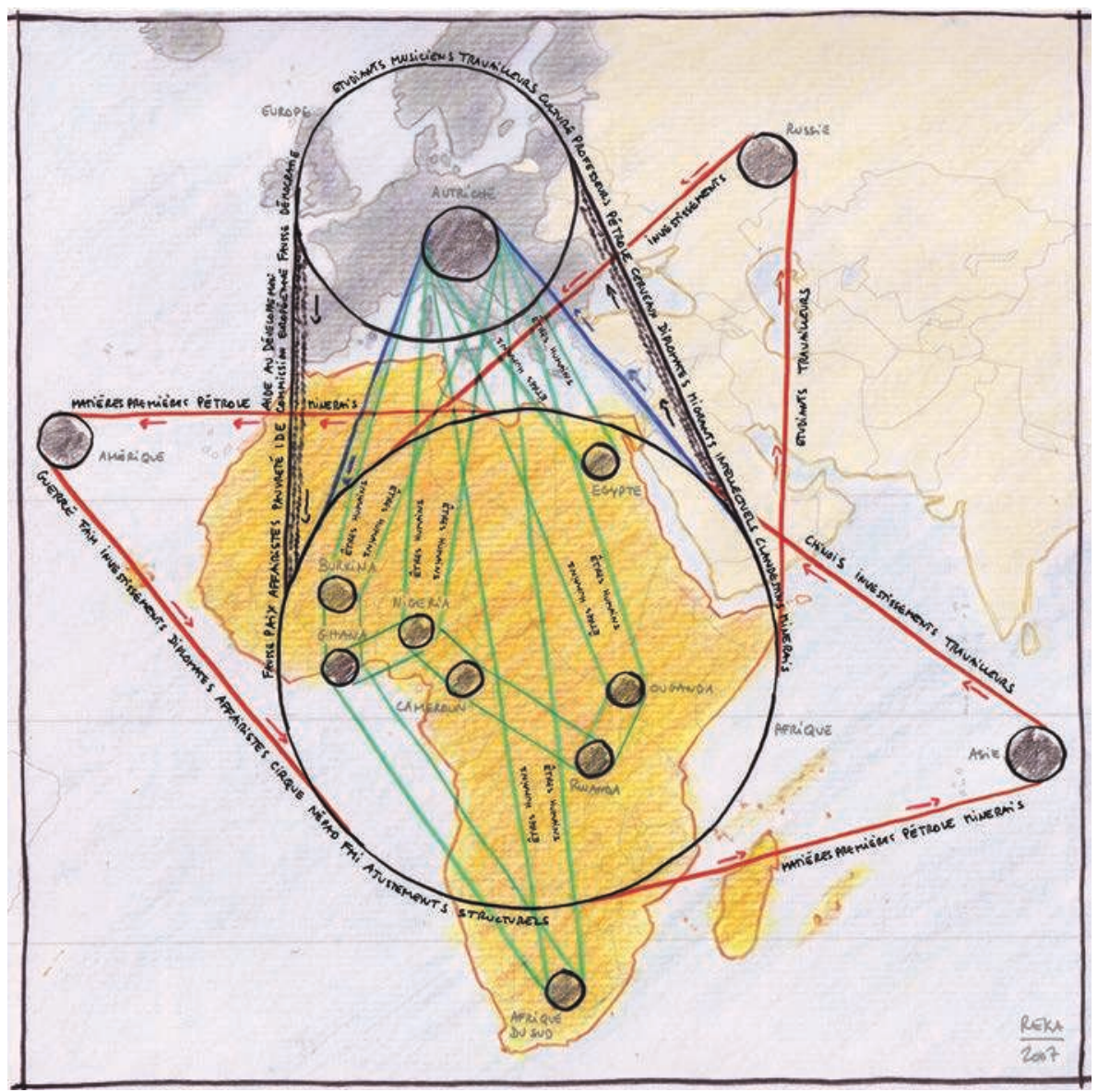

Crédit : P. Rekacewicz, 2007. 
À titre d'exemple, la carte 10 de la "Grande roue africaine ", dessinée à la main, entre ambition scientifique et artistique, place les questions migratoires dans un système de causalités géopolitiques et économiques, existant au sein du continent et entre l'Afrique et les autres continents. La carte figure un système d'échange assez complexe dans lequel le continent africain est littéralement "empêtré ». Elle décrit un mouvement perpétuel entre I'Afrique et différents partenaires, que les flèches ne symboliseraient que très imparfaitement. Le rouage, mode de représentation continu, symbolise la permanence et la continuité dans les termes d'un échange profondément inégal : "I'Afrique sauve I'Europe qui appauvrit l'Afrique qui nourrit l'Europe qui asservit l'Afrique qui paye l'Europe qui continue de détrousser l'Afrique... ". Cette symbologie circulaire peut être considérée comme une forme de représentation alternative à la flèche.

Les cartes présentées dans la section suivante, plutôt que donner à voir des systèmes de causalité et des dispositifs de contrôle, relatent plus spécifiquement des expériences individuelles et collectives, en proposant d'adopter le point de vue de celles et ceux qui se déplacent.

\section{Cartographies critiques des expériences migratoires}

Les cartes présentées ici traitent de ce que les trajectoires vécues engagent en termes d'obstacles et d'opportunités, de contraintes ou de ressources, de pratiques et d'imaginaires.

Les cartographies manuscrites de parcours migratoires individuels réalisées dans le cadre de l'exposition "Moving Beyond Borders " ${ }^{36}$ en sont un exemple. Associées en binôme à des cartes numériques sur les dispositifs de contrôle, ces cartes manuscrites ont pour objectif de représenter leurs effets sur le parcours et le vécu des individus. C'est le cas de la carte dessinée par Lucie Bacon et intitulée "Cinq ans pour rejoindre Hambourg depuis Kaboul ", réalisée sur la base d'un entretien entre un citoyen afghan rencontré en Roumanie, et Bénédicte Michalon, chercheuse et membre de Migreurop. La carte exprime le poids de l'enfermement, les temporalités et les espaces parcourus, les mobilités volontaires et contraintes, les expériences multiples de privation de liberté (cf. Carte 11).

Dans le registre de la visualisation de données narratives et discursives, I'œuvre cartographique " H-OUT-Guide de l'immigration ", réalisée en 2010 par le graphiste et illustrateur algérien Zineddine Bessaï, a été conçue comme un guide cartographié à l'usage des harragas ${ }^{37}$. La carte présente la Méditerranée et ses deux rives, avec de nombreux symboles représentant les dispositifs de contrôle, mais aussi des figures de récits entendus depuis les rives du Maghreb. Les toponymes sont des translittérations de l'arabe et reprennent le vocabulaire lié à la harraga : par exemple, l'Europe est traduite par "Oropa ", et la mer Méditerranée, par " la mort méditerranée ".

36 Moving Beyond Borders est une exposition itinérante produite par Migreurop et mise en scène par la compagnie Étrange Miroir.

37 Terme en arabe maghrébin qui signifie les "brûleurs ", celles et ceux qui "brûlent " les frontières. Ainsi, les " harragas " désignent les migrant.e.s qui tentent de franchir la mer Méditerranée depuis les rives du Maghreb. 
Carte 11 : Extrait de la carte intitulée « 5 ans pour rejoindre Hambourg depuis Kaboul »

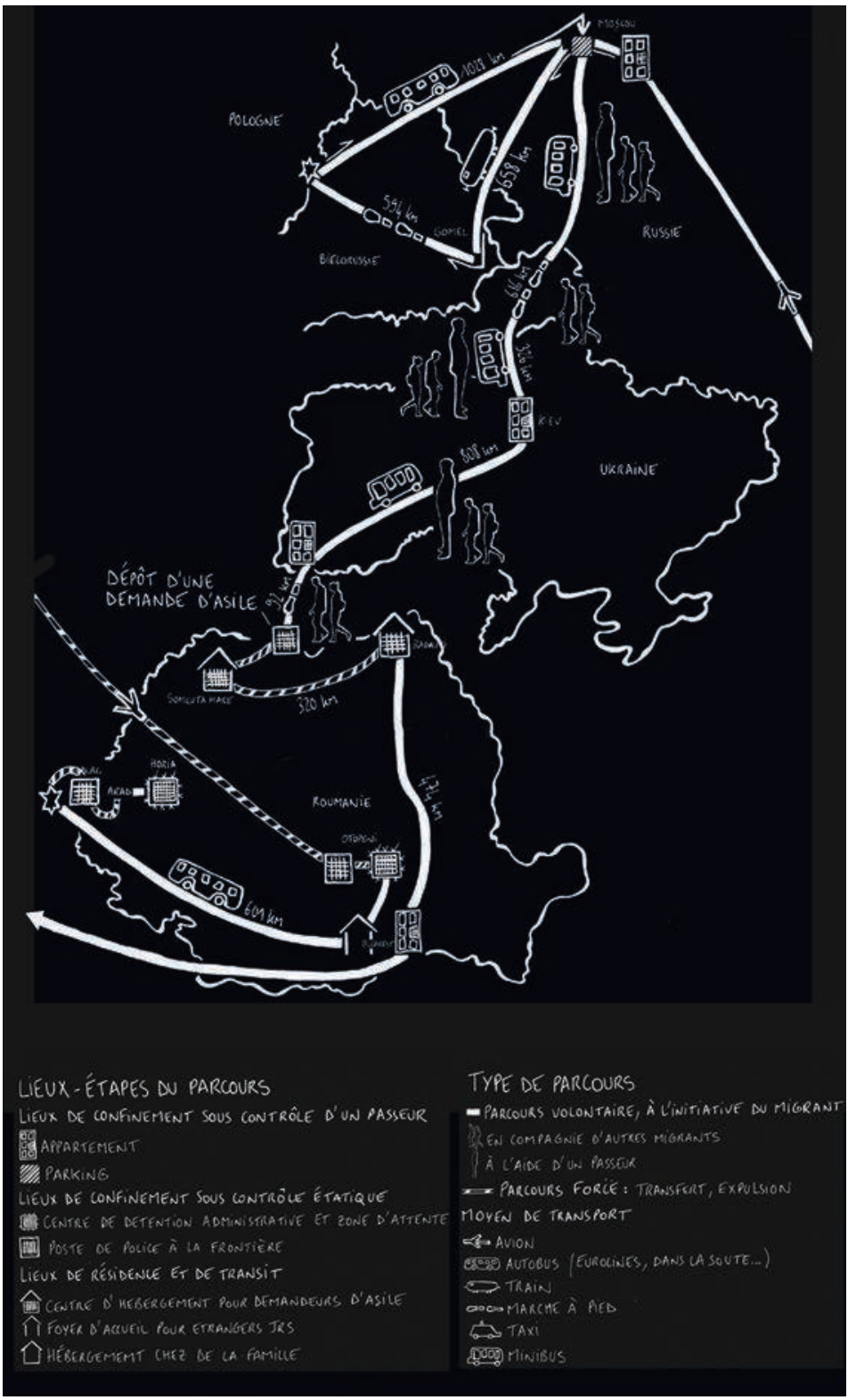

Source : Michalon Bénédicte (2010) Entretien réalisé à Bucarest. Crédit : L. Bacon, 2015. 
« The Mapping Journey Project ", installation réalisée entre 2008 et 2011 par Bouchra Khalili, artiste franco-marocaine, s'inscrit également dans un processus de cartographies narratives. Composée de huit courts-métrages (The Mapping Journey) et de huit sérigraphies (The Constellations), cette installation donne à voir et à entendre des récits de parcours migratoires individuels. Chaque vidéo est un plan fixe où se meut une main traçant un parcours sur un fond de carte conventionnel. L'image est accompagnée de la voix de la personne qui dessine et qui élabore un récit à l'oral. Les sérigraphies (issues de huit récits, réalisés dans cinq pays et six villes différents) cartographient des trajectoires sous forme de constellations d'étoiles blanches sur fond bleu uni, en brouillant ainsi les repères topographiques conventionnels (cf. Carte 12).

Carte 12 : The Constellations (Figure 8 de la série The Constellations, 8 sérigraphies, $60 \times 40 \mathrm{~cm}, 2011$ )

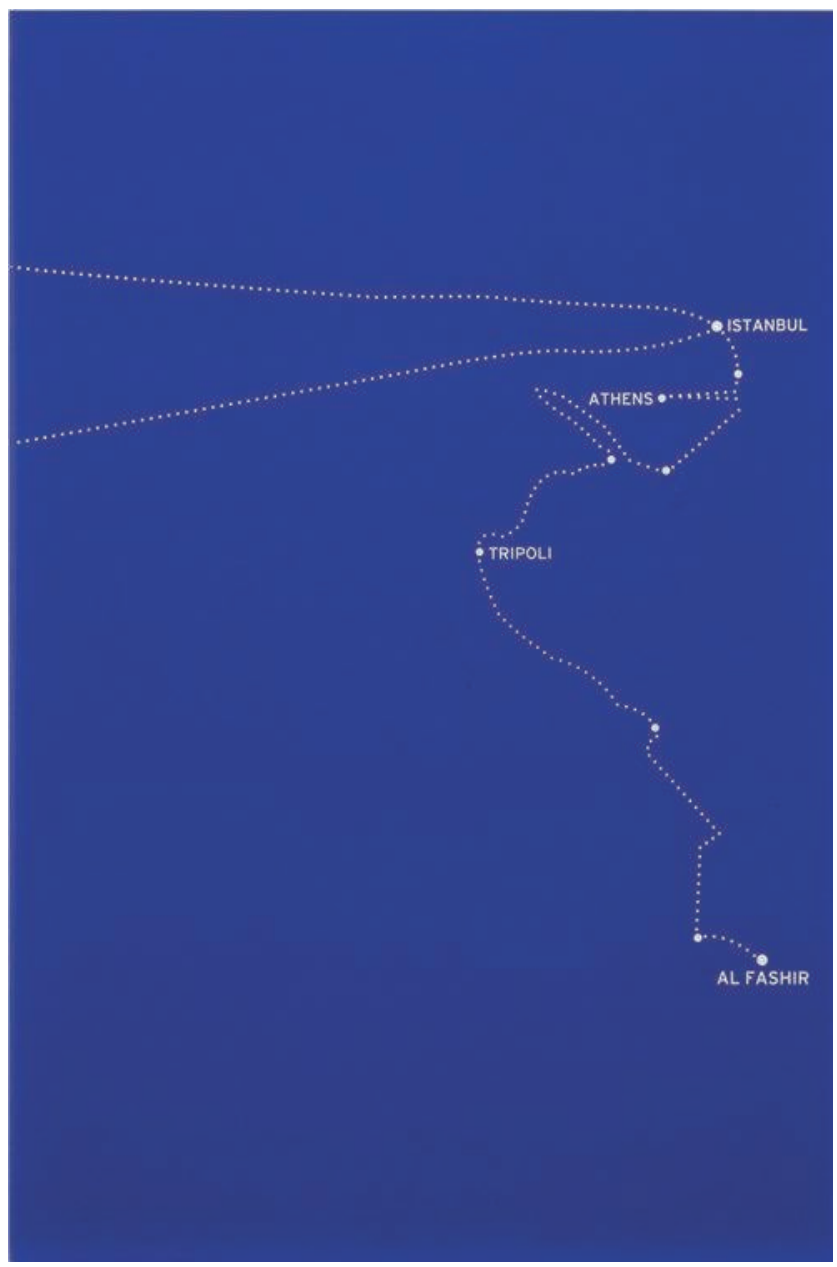

Source : The Opposite of The Voice-Over, exposition personnelle, Färgfabriken Konsthall, Stockholm, 2016. 
Au-delà du processus de ré-énonciation cartographique de récits et de discours entendus ou provoqués par les chercheur.se.s, les travaux suivants sont issus de coproductions entre personnes exilées, artistes et/ou chercheur.se.s.

"Latino/a » est une série cartographique réalisée par I'artiste mexicain Pedro Lasch en 2003 et huit personnes ayant traversé la frontière entre le Mexique et les États-Unis. Ce travail met en jeu la carte depuis une perspective " plusque-représentationnelle ". Autrement dit, l'intérêt est porté à ce que représente la carte, mais aussi à la carte comme objet transporté pendant les voyages. De 2003 à 2006, l'artiste a remis quarante cartes représentant l'Amérique du Nord à vingt personnes qui se préparaient à traverser la frontière nord du Mexique vers les États-Unis. Chacune de ces personnes a reçu deux cartes : une première à conserver et une seconde à renvoyer à l'artiste à l'arrivée. Cette installation rassemble les huit cartes que Pedro Lasch a reçues. Froissées, pliées, décolorées, elles portent les marques de la traversée. Chacune d'entre elles est accompagnée d'un cartel indiquant le nom de la personne qui en est la ou le coauteur.e, accompagné de quelques éléments relatifs à son expérience.

Enfin, I'installation plastique "Cartographies traverses / Crossing Maps " relève d'un processus créatif partagé. Cette installation a été produite en 2013 par des chercheuses des artistes et douze habitant.e.s grenoblois.e.s en situation de demande d'asile ou de réfugié.e. ${ }^{38}$. Réuni.e.s en atelier, les coauteurs.e.s ont réalisé une légende collective à partir de mots significatifs des expériences migratoires. Les mots ont été symbolisés par des gommettes de formes et de couleurs différentes. À partir de ce travail, qui avait notamment pour intention de dépasser l'atomisation de la parole individuelle, chaque participant.e a dessiné plusieurs cartes utilisant la légende collective. Certain.e.s ont aussi modelé leur carte dans l'argile ou dessiné et brodé sur de grandes nappes blanches, à l'invitation de Marie Moreau, artiste-plasticienne. Ce travail est autant une recherche sur les modalités possibles de relations à établir entre artistes, chercheuses et personnes en situation de lutte pour leurs droits, que sur les modes de figuration des expériences migratoires (cf. Carte 13).

38 Marie Moreau, Lauriane Houbey, Fabien Fischer, artistes, Sarah Mekdjian et AnneLaure Amilhat Szary, géographes, Laetitia Abbas, Alishum Ahmedin, Ahmedin A., Shamil Astahanov, Nasruddin Gladeema, Karim huseynov, Issa Ibrahm Hamid, Fiston Massamba, Tatevik Melkonyan, Salomon Paluku, Aboubakar Souleiman Guelleh, Kanké Tounkara, habitant.e.s grenoblois.e.s, en situation de réfugié.e.s ou de demandeur.se.s d'asile. 


\section{Carte 13 : The World is Stopping Us}

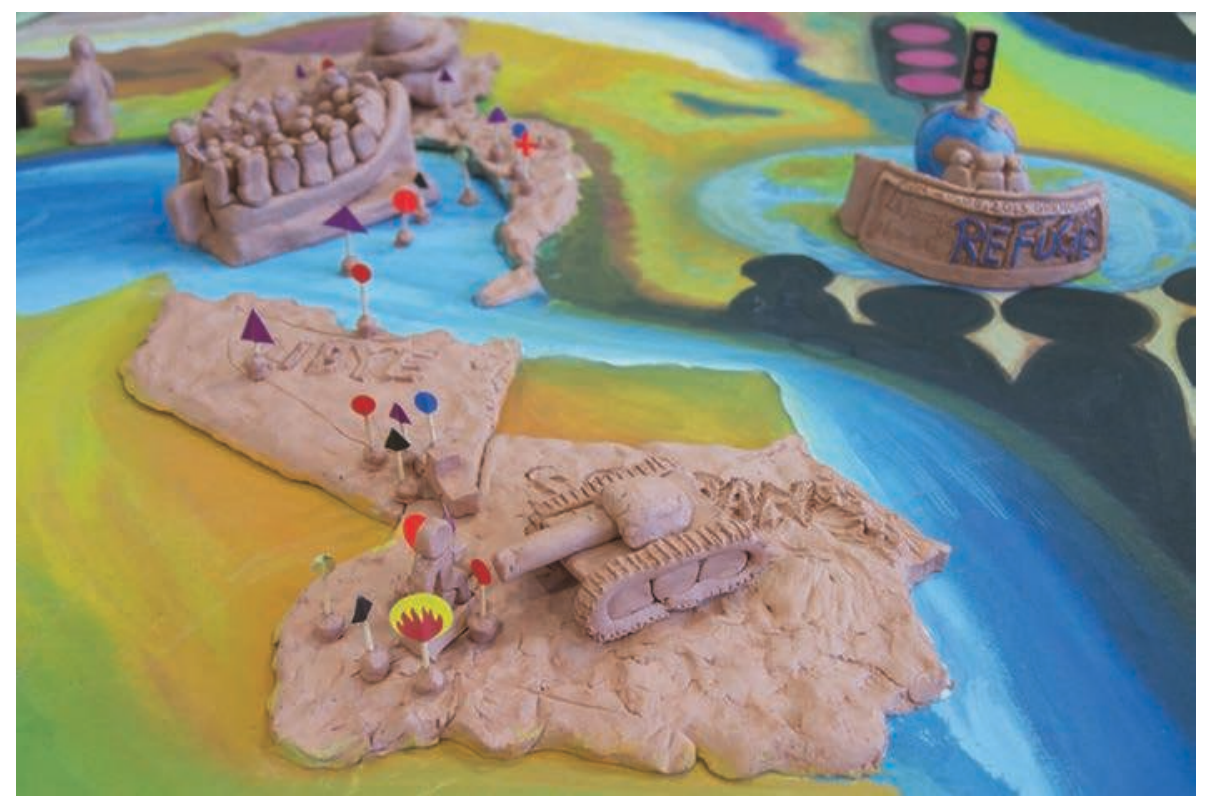

Source : Gladeema Nasruddin (2013) Ateliers de cartographie à Grenoble.

Crédit : Mabeye Deme, 2013.

\section{Conclusion}

Au même titre que la recherche en migrations internationales s'est dotée de nouveaux cadres théoriques et conceptuels pour penser les mobilités dans leur changement et leur complexification, les cartographies semblent être elles aussi remises en cause dans leurs fondements par les dynamiques internationales. Les migrations réinterrogent non seulement le territoire et les frontières dans leur conception classique, mais elles conduisent également à faire émerger d'autres types de représentations de l'espace, du mouvement et des enjeux politiques associés.

Une partie des cartes présentées, en particulier dans la dernière partie de l'article, ont pour point commun d'associer (et d'assumer) des intentions artistiques, politiques, scientifiques et militantes. On pourrait les définir comme relevant de cartographies " loin des us et coutumes académiques " et qui s'affirment « contre la pensée unique " (Lambert, 2013).

Néanmoins, ces cartes ne peuvent pas se penser uniquement comme des " contre-cartographies " : plutôt que de les opposer aux cartes qui répondent à un régime normé de représentation (flèches par exemple), il s'agit de les penser comme complémentaires. Depuis la posture du dévoilement et de la dénonciation, jusqu'au processus de subjectivation du geste et de la figuration cartographique, ces réalisations entendent dénaturer nos imaginaires des migrations, largement construits à partir d'images récurrentes et omniprésentes, et notamment cartographiques. 


\section{Références bibliographiques}

Amilhat-Szary Anne-Laure, Fischer Fabien, Houbey Lauriane, Mekdjian Sarah, Moreau Marie et douze habitant.e.s grenoblois.e.s (2013) Cartographies traverses/ Crossing Maps, [en ligne]. URL : http://www.antiatlas.net/blog/2013/09/20/crossing-maps-cartographies-traverses/

Avelot René (1905) Ethnographie et migrations au Gabon et au Congo Français, $1 / 4000$ 000, [en ligne]. URL : http://gallica.bnf.fr/ark:/12148/btv1b8444725n/ f1. item. $r=$ congo.langEN

Bahoken Françoise (2013) Sur la première carte des flux réalisée avec des flèches (Ravenstein, 1885), Confins (revue franco-brésilienne de géographie/revista franco-brasilera de geografia), 17, [en ligne]. URL : http://confins.revues.org/8187

Bahoken Françoise (2012) Contribution du raisonnement logique à la cartographie des flux, in Rolland Billen, Marc Binard, Pierre Hallot et Jean-Paul Donnay, Actes de la Conférence internationale de Géomatique et Analyse Spatiale, Université de Liège (6 au 9 novembre 2012), pp. 13-29, [en ligne]. URL : https:// orbi.ulg.ac.be/bitstream/2268/133126/1/PROCEEDINGS_A4.pdf

Bahoken Françoise (2011) Représentation graphique des matrices. Graphe et/ou carte des flux ?, 13 p. [en ligne]. URL : https://halshs.archives-ouvertes.fr/halshs00641733/document

Bahoken Françoise (2009a) Les documents cartographiques en ligne sur les migrations internationales, e-migrinter, 3, pp. 37-52, [en ligne]. URL : http://migrinter.labo.univ-poitiers.fr/wp-content/uploads/sites/150/2016/05/ emigrinter2009_03_tout.pdf

Bahoken Francoise (2009b) Atlas sur les migrations internationales, e-migrinter, 3, pp. 53-57, [en ligne]. URL : http://migrinter.labo.univ-poitiers.fr/wp-content/ uploads/sites/150/2016/05/emigrinter2009_03_tout.pdf

Béguin Michèle et Pumain Denise (2007 [réédition]) La représentation des données géographiques : statistique et cartographie, Paris, Armand Colin, 192 p.

Bertin Jacques (1967 [1 ère éd.]) Sémiologie graphique. Les diagrammes, les réseaux, les cartes, Paris, La Haye, Mouton, Gauthier-Villars, 452 p.

Bessaï Zineddine (2010) H-OUT-Guide de l'immigration, [en ligne]. URL : http:// zineddinebessai.com/portfolio/h-out/

Boltanski Luc (2007) La souffrance à distance, Paris, Folio Essais, 528 p.

Boyer Florence (2005) Être migrant et Touareg de Bankilaré (Niger) à Abidjan (Côte d'Ivoire) : des parcours fixes, une spatialité nomade, Thèse en géographie, Université de Poitiers, $578 \mathrm{p}$.

Brunet Roger (1987) La carte mode d'emploi, Paris, Fayard, 270 p.

Choplin Armelle et Pliez Olivier (2011) De la difficulté de cartographier l'espace saharo-sahélien, $M @$ ppemonde, 103, [en ligne]. URL : http://mappemonde.mgm. fr/num31/intro/intro2.html

Clochard Olivier et Honoré Thomas (2015) Représenter des flux migratoires : un défi cartographique, Carto, 31, pp. 54-56.

Clochard Olivier and Lambert Nicolas (2015) Mobile and Fatal:The EU Borders, in Anne-Laure Amilhat Szary and Frédéric Giraut Eds, Borderities and the Politics of Contempory Mobile Borders, New-York, Palgrave McMillan, pp. 119-137. 
Cosgrove Denis (2001) Apollo's Eye: A Cartographic Genealogy of the Earth in the Western Imagination, Baltimore, The Johns Hopkins University Press, 352 p.

Crampton Jeremy W. (2009) Cartography: Performative, Participatory, Political, Progress in Human Geography, 33 (6), pp. 840-848.

Crampton Jeremy W. and Krygier John (2005) An introduction to Critical Cartography, ACME-An International E-Journal for Critical Geographies, 4 (1), pp. 11-33, [online]. URL: http://acme-journal.org/index.php/acme/article/view/723

Cvijić Jovan (1918) Courants métanostasiques dans le peuplement des pays serbes du XVe siècle à nos jours, Paris, Armand Colin, [en ligne]. URL : http:// gallica.bnf.fr/ark:/12148/btv1b53060979d. $r=$

Delage Cécile et Miellet Philippe (2001) Représentation cartographique : guide méthodologique, Paris Certu, Gip Aten, 87 p.

Forensic Architecture (2011) The Let-to-Die Boat, [online]. URL: http://www. forensic-architecture.org/case/left-die-boat/

Gintrac Cécile (2012) Géographie critique, géographie radicale : Comment nommer la géographie engagée?, Carnets de géographes, 4, [en ligne]. URL: http://www.carnetsdegeographes.org/carnets_recherches/rech_04_04_Gintrac. php

Gould Peter et Bailly Antoine (1995) Le pouvoir des cartes, Brian Harley et la cartographie, Paris, Economica, 120 p.

Hackitectura (2004) Cartografía crítica del estrecho de Gibraltar, [en línea]. URL: http://dunadigital.com/blog/2004/07/11/cartografia-critica-del-estrecho-degibraltar-fadaiat-2004/

Heller Charles et Pezzani Lorenzo (2014) Traces liquides : enquête sur la mort de migrants dans la zone-frontière maritime de I'Union européenne, Revue Européenne des Migrations Internationales, 30 (3), pp. 71-107, [en ligne]. URL : http://remi.revues.org/7106

Jacob Christian (1992) L'Empire des cartes: Approche théorique de la cartographie à travers l'histoire, Paris, Albin Michel, $532 \mathrm{p}$.

Kaddouri Lahouri (2008) Réflexion sur la sémiologie graphique animée des flux, Mappemonde, 89, [en ligne]. URL : http://mappemonde.mgm.fr/num17/articles/ art08104.html

Khalili Bouchra (2011) The Mapping Journey Project, [online]. URL: http://www. bouchrakhalili.com/the-mapping-journey-project/

Lacoste Yves (1976) La géographie, ça sert, d'abord, à faire la guerre, Paris, Maspéro, 248 p.

Lambert Nicolas (2013) Cartographie radicale, Carnet Neocartographique, [en ligne]. URL : http://neocarto.hypotheses.org/15

Lambert Nicolas (depuis 2010) Carnet Néocartographique, [en ligne]. URL : http:// neocarto.hypotheses.org/

Lasch Pedro (2006) Latino/a, [online]. URL: http://www.pedrolasch.com/latinoaamerica.html

Lessault David et Beauchemin Cris (2009) Ni invasion, ni exode, Revue Européenne des Migrations Internationales, 25 (1), [en ligne]. URL : http://remi.revues.org/4889 
Lévy Jacques, Poncet Patrick et Tricoire Emmanuelle (2004) La carte, enjeu contemporain, La documentation photographique, 8036, 63 p.

Liagre Romain (2008) De la difficulté de cartographier les itinéraires migratoires clandestins, Espace populations sociétés, 3, pp. 453-461, [en ligne]. URL : http:// eps.revues.org/3520

Mekdjian Sarah (2015) Mapping Mobile Borders. Critical cartographies of borders based on migration experiences, in Anne-Laure Amilhat Szary and Frédéric Giraut Eds., Borderities and the Politics of Contemporary Mobile Borders, New-York, Palgrave McMillan, pp. 204-224.

Mekdjian Sarah, Amilhat-Szary Anne-Laure, Moreau Marie, Nasruddin Gladeema, Deme Mabeye, Houbey Auriane et Guillemin Coralie (2014) Figurer les entredeux migratoires : Pratiques cartographiques expérimentales entre chercheurs, artistes et voyageurs, Carnets de géographes, 7, [en ligne]. URL : http://www. carnetsdegeographes.org/carnets_terrain/terrain_07_01_Mekdjian.php

Migreurop (depuis 2013) Closethecamps, [en ligne]. URL : http://closethecamps. org/

Migreurop (2012) Atlas des migrants en Europe. Géographie critique des politiques migratoires, Paris, Armand Colin, $144 \mathrm{p}$.

Migreurop et Étrange Miroir (2015) Moving Beyond Borders, [en ligne]. URL : http://www.migreurop.org/article2601.html

Mitchell William John Thomas (1995) Interdisciplinarity and Visual Culture, Art Bulletin, 77 (4), pp. 540-544.

Poulain Michel (1992) Un projet d'harmonisation des statistiques de migration internationale au sein de la Communauté Européenne, Revue Européenne des Migrations Internationales, 8 (1), pp. 163-186.

Rekacewicz Philippe (2014) Entre imaginaire et réalité : I'intention cartographique, Revue 303, 133, pp. 8-15.

Rekacewicz Philippe (2013) Cartographie radicale, Le Monde diplomatique, février, p. 15 [en ligne]. URL : http://www.monde-diplomatique.fr/2013/02/ REKACEWICZ/48734

Rekacewicz Philippe (2006) La cartographie, entre science, art et manipulation, Le Monde diplomatique, février, pp. 14-15, [en ligne]. URL : http://www.mondediplomatique.fr/2006/02/REKACEWICZ/13169

Rekacewicz Philippe et Rivière Philippe (depuis 2014) Visionscarto, [en ligne]. URL : http://visionscarto.net/

Robin Nelly (2014) Migrations, observatoire et droit. Complexité du système migratoire ouest-africain. Migrants et normes juridiques, Habilitation à diriger des recherches, vol. 1, Université de Poitiers, 143 p.

Robinson Arthur H. (1955) The 1837 maps of Henry Drury Harness, The Geographic Journal, 121 (4), pp. 440-450.

Schrader Franz et Gallouédec Louis (1923) Atlas classique de géographie ancienne et moderne, Paris, Hachette.

Simon Gildas (2009) Migrations, la spatialisation du regard, Revue Européenne des Migrations Internationales, 22 (2), pp. 9-21, [en ligne]. URL : http://remi. revues.org/2815 
Simon Gildas (2008) La planète migratoire dans la mondialisation, Paris, Armand Colin, $255 \mathrm{p}$.

Simon Gildas (1992) Une Europe Communautaire de moins en moins mobile ?, Revue Européenne des Migrations Internationales, 8 (1), pp. 67-87, [en ligne]. URL : http://www.persee.fr/doc/remi_0765-0752_1992_hos_8_1_1034

Transit Migration (2005) Migmap, [online]. URL: http://www.transitmigration.org/ migmap/

Wood Michael (2004) La cartographie est éternelle, Géomatique Expert, 34, pp. 19-26, [en ligne]. URL : http://www.geomag.fr/rev/pdf/34_34.pdf 


\section{Lucie Bacon, Olivier Clochard, Thomas Honoré, Nicolas Lambert, Sarah Mekdjian et Philippe Rekacewicz}

\section{Cartographier les mouvements migratoires}

À partir de diverses réalisations conventionnelles en géographie et/ou artistiques, parmi lesquels des travaux réalisés par les auteurs, cet article s'intéresse à la manière dont la cartographie des migrations internationales a évolué depuis le début des années 1990. La représentation des mouvements migratoires, qui oscille entre des flèches, des mesures de stocks et diverses formes de dessin, soulève autant d'enjeux scientifiques et politiques posés à la cartographie et géographie des migrations. Après un bref rappel des changements qu'a connus la cartographie des flux migratoires, depuis ses origines, au XIXe siècle, jusqu'à aujourd'hui, les auteurs évoquent les principaux défis techniques et épistémologiques que les cartes de flux ou représentant des itinéraires ne cessent de poser. Puis pour terminer, sont présentées de nouvelles formes cartographiques, ayant émergé depuis le début des années 2000, dans les domaines croisés de la science, de l'art et du militantisme.

\section{Mapping the Migratory Movements}

Based on several conventional geography works and/or artistic works - some of them made by the authors of this paper -, this article focuses on how the mapping of international migrations has evolved since the beginning of the 1990s. The representation of migratory movements, which oscillates between arrows, measurements of stocks and different forms of design, implies both scientific and political stakes for the cartography and geography of migrations. After making a brief recalling of the changes that the mapping of migratory flows has experienced from its origins in the 19th century until today, the authors refer to the main technical and epistemological challenges that cartography of migratory movements and the presentations of itineraries raise nowadays. Finally the article analyses the new cartographic forms that have emerged since the beginning of the 2000s within the interconnected domains of sciences, arts, and militancy.

\section{Cartografiar los movimientos migratorios}

Partiendo de diversos trabajos convencionales de geografía y/o artísticos algunos de ellos realizados por los propios autores -, este artículo pretende mostrar cómo la cartografía de las migraciones internacionales ha ido evolucionando desde principios de los años 1990. La representación de los movimientos migratorios, que abarca desde flechas, hasta medidas de reserva y diferentes formas de diseño, supone para la cartografía y la geografía de las migraciones toda una serie de retos, tanto científicos como políticos. Tras hacer un repaso de los cambios que ha ido experimentando la cartografía de los flujos migratorios desde sus orígenes en el siglo XIX hasta la actualidad, los autores presentan los principales retos técnicos y epistemológicos que los mapas de flujos y las representaciones de itinerarios continúan planteando en el momento actual. Por último, se presentan nuevas formas cartográficas surgidas a partir del 2000 que se inscriben en ámbitos interconectados como la ciencia, el arte y el activismo. 